\title{
The molecular circumnuclear disk (CND) in Centaurus A
}

\section{A multi-transition $\mathrm{CO}$ and [CI] survey with Herschel, APEX, JCMT, and SEST}

\author{
F. P. Israel ${ }^{1}$, R. Güsten ${ }^{2}$, R. Meijerink ${ }^{3}$, A. F. Loenen ${ }^{1}$, M. A. Requena-Torres ${ }^{2}$, J. Stutzki ${ }^{4}$, P. van der Werf ${ }^{1}$, \\ A. Harris $^{5}$, C. Kramer ${ }^{6}$, J. Martin-Pintado ${ }^{7}$, and A. Weiss, ${ }^{2}$ \\ ${ }^{1}$ Sterrewacht Leiden, Leiden University, PO Box 9513, 2300 RA Leiden, The Netherlands \\ e-mail: israel@strw.leidenuniv.nl \\ 2 Max-Planck-Institut für Radioastronomie, Auf dem Hügel 69, 53121 Bonn, Germany \\ 3 Kapteyn Astronomical Institute, Postbus 800, 9700 AV Groningen, The Netherlands \\ ${ }^{4}$ I. Physikalisches Institut der Universität zu Köln, Zülpicher Strasse 77, 50937 Köln, Germany \\ 5 Department of Astronomy, University of Maryland, College Park, MD 20742, USA \\ 6 IRAM, Avenida Divina Pastora, 7, Núcleo Central, 18012 Granada, Spain \\ 7 CSIC/INTA, Ctra de Torrejón a Ajalvir, km 4, 28850 Torrejón de Ardoz, Madrid, Spain
}

Received 2 October 2013 / Accepted 16 December 2013

\begin{abstract}
This paper presents emission line intensities of $\mathrm{CO}$ and $\mathrm{C}^{\circ}$ from the compact circumnuclear disk in the center of NGC 5128 (Centaurus A) obtained with the Herschel Space Observatory in the 400-1000 GHz range as well as previously unpublished measurements obtained with the ground-based observatories SEST, JCMT and APEX in the 90-800 GHz range. The results show that the Cen A center has an emission ladder of $\mathrm{CO}$ transitions quite different from those of either star-burst galaxies or (Seyfert) AGNs. In addition, the neutral carbon ([CI]) emission lines from the Cen A center are much stronger relative to the adjacent CO lines than in any other galaxy. The CO surface brightness of the compact circumnuclear disk (CND) is significantly higher than that of the much more extended thin disk (ETD) in the same line of sight. LVG analysis of the CO line profiles decomposed into the constituent contributions show that the ETD is relatively cool and of low excitation, wheres the brighter CND is hotter and more highly excited. Our PDR/XDR models suggest that most of the CND gas is relatively cool (temperatures $25 \mathrm{~K}-80 \mathrm{~K})$ and not very dense $\left(\approx 300 \mathrm{~cm}^{-3}\right)$ if it is primarily heated by UV photons. A small fraction of the gas in both the CND and the ETD has a much higher density (typically $30000 \mathrm{~cm}^{-3}$ ). A more highly excited, high-density phase is present in the CND, either in the form of an extreme PDR or more likely in the form of an XDR. Such a phase does not occur in the part of the ETD sampled. We have determined, for the first time, the molecular mass parameters of the CND. The total gas mass of the CND is $M_{\mathrm{CND}}=8.4 \times 10^{7} M_{\odot}$, uncertain by a factor of two. The CO- $\mathrm{H}_{2}$ conversion factor $\left(X_{\mathrm{CND}}\right)$ is $4 \times 10^{20}\left(\mathrm{~K} \mathrm{~km} \mathrm{~s}^{-1}\right)^{-1}$ also within a factor of two.
\end{abstract}

Key words. ISM: molecules - galaxies: active - galaxies: elliptical and lenticular, cD - galaxies: individual: Centaurus A galaxies: ISM - galaxies: nuclei

\section{Introduction}

Disks of dense dust and gas deeply embedded in the stellar body of a giant elliptical galaxy are the more easily identified remnants of smaller gas-rich galaxies that have fallen in. They are a transient phenomenon: on varying time-scales, the gas will be consumed by accretion onto a central black hole, expulsion by jets emanating from the nucleus, and by the in-situ formation of new stars rejuvenating the host galaxy stellar population. All three processes are currently active in NGC 5128, the host of the Fanaroff-Riley class I (FR I) radio source Centaurus A (Cen A).

The properties of these embedded disks are probably different from those of the interstellar medium (ISM) in spiral or starburst galaxies (see e.g. the review by Henkel \& Wiklind 1997). Kiloparsec-sized embedded thin disks are directly exposed to the intense but UV-poor combined radiation from all stars in the host elliptical. There is little differential rotation, and the lack of shear may locally favour the formation of massive groups of luminous stars in the disk affecting their surrounding ISM. On much smaller sub-kiloparsec scales, the dynamics of a denser circumnuclear disk may be only loosely related to those of the larger kiloparsec-sized extended disk. Instead, in such a circumnuclear disk both dynamics and excitation expected to be tied more closely to the properties of the super-massive black hole in the nucleus.

The emission lines from carbon and carbon monoxide are key to understanding the properties of the ISM in galaxies, as they provide almost all the cooling of the dense neutral gas. Warm and tenuous gas is traced by ionised carbon ([C II] ), warm and dense gas by neutral carbon ([C I] ) and carbon monoxide $(\mathrm{CO})$, and cold and dense gas by $(\mathrm{CO})$ and its isotopologues. Although the two neutral carbon [C I] lines at 492 and $809 \mathrm{GHz}$ and $\mathrm{CO}$ lines with rest frequencies up to about $1000 \mathrm{GHz}$ can be measured from the ground (albeit with increasing difficulty), the ionised carbon [C II] fine-structure line at $1.9 \mathrm{THz}$ requires a platform above most or all of the atmosphere.

NGC 5128 is the nearest $(D=3.84 \mathrm{Mpc})$ giant elliptical (review by Israel 1998). Its central black hole is still accreting in the aftermath of a merger with a medium-sized late-type galaxy a few hundred million years ago (Graham 1979; Struve et al. 2010). The remnant ISM of the merged galaxy has redistributed itself into a warped, thin disk ("the extended thin 
disk" - hereafter called the ETD - which shows in projection as a dark band crossing the optical image of the galaxy (Dufour et al. 1979; Nicholson et al. 1992). It has comparable amounts of atomic ( $\mathrm{H} \mathrm{I}$ ) and molecular $\mathrm{H}_{2}$ gas, and its total mass is uncertainly estimated at $1.5 \times 10^{9} M_{\odot}$, about two per cent of the enclosed dynamical mass. On significantly smaller scales, the nuclear black hole of $5 \times 10^{7} M_{\odot}$ (Cappellari et al. 2009) is obscured by a compact circumnuclear disk (CND) that feeds the black hole (Israel et al. 1990).

Unlike the ETD, the compact CND is oriented at right angles to the luminous radio and X-ray jets emanating from the Centaurus A nucleus. This suggests that the CND and the jets are somehow connected, but it is not yet clear how that may be. The CND is a well-defined entity with a diameter of 20" (400 pc). It has been seen in FIR continuum (Hawarden et al. 1993) and ${ }^{12} \mathrm{CO}$ line emission (Espada et al. 2009), but was not yet studied in detail.

In this paper, we combine single-dish $\mathrm{CO}$ and $[\mathrm{CI}]$ observations of the Cen A nucleus obtained from the ground over many years, and from space. Although observations of the Cen A nucleus distinguishing CND and ETD in the lower $\left(J_{\text {upper }} \leq 3\right)$ transitions of $\mathrm{CO}$ are found in a few earlier papers (Israel et al. 1990; Israel 1992; Rydbeck et al. 1993), all data in this paper have much higher signal-to-noise ratios and are presented here for the first time.

\section{Observations and data handling}

\subsection{Herschel-SPIRE}

The nucleus of Centaurus A was observed with the Spectral and Photometric Imaging Receiver and Fourier-Transform Spectrometer (SPIRE-FTS - Griffin et al. 2010) onboard the Herschel Space Observatory ${ }^{1}$ (Pilbratt et al. 2010) in the singlepointing mode with sparse image sampling. The FTS has two detector arrays (the SLW: wavelength range 303-671 $\mu \mathrm{m}$ corresponding to a frequency range 447-989 GHz, and the SWW: wavelength range $194-313 \mu \mathrm{m}$ corresponding to a frequency range 959-1544 GHz). The observations are summarised in Table 1 . The data were processed and calibrated using HIPE version 9.0.0. The spectral range covered the ${ }^{12} \mathrm{CO}$ lines in the $J=4-3$ to $J=13-12$ transitions as well as the two submillimeter [C I] lines, which were all detected; however, no ${ }^{13} \mathrm{CO}$ lines were detected. The spectral resolution of $1.21 \mathrm{GHz}$ was insufficient to resolve any of the lines in the SLW spectrum, but may start to resolve some of the lines in the SSW spectrum, particularly the [N II] line. Line fluxes were extracted by fitting a sinc-Gaussian function to the line profile. The beam FWHM values are given in the on-line Herschel-SPIRE manual; they range from $29^{\prime \prime}$ to $42^{\prime \prime}$ for the SLW, and from $16.8^{\prime \prime}$ to $21.1^{\prime \prime}$ for the SSW. At the overlap between the two arrays, at about $310 \mu \mathrm{m}$ or $964 \mathrm{GHz}$, the beam size jumps from 21.1" to $37.3^{\prime \prime}$ going from SSW to SLW. The results are shown in Fig. 3, and summarised in Tables 2 and 4.

\subsection{Herschel-HIFI}

The center of Centaurus A was also observed with the Heterodyne Instrument for the Far Infrared (HIFI - de Graauw et al. 2010) on-board Herschel as part of the guaranteed time

\footnotetext{
1 Herschel is an ESA space observatory with science instruments provided by European-led Principal Investigator consortia and with important participation from NASA.
}

Table 1. Log of Herschel observations.

\begin{tabular}{|c|c|c|c|c|}
\hline Instrument & Transition & OBSID & $\begin{array}{c}\text { Date } \\
\text { Y-M-D }\end{array}$ & $\begin{array}{r}\text { Integr. } \\
\text { (s) }\end{array}$ \\
\hline HIFI & $\mathrm{CO} J=5-4 \mathrm{C}$ & 1342200931 & $2010-07-19$ & 1863 \\
\hline HIFI & $\mathrm{CO} J=5-4 \mathrm{NW}$ & 1342200932 & 2010-07-19 & 86 \\
\hline HIFI & $\mathrm{CO} J=5-4 \mathrm{SE}$ & 342200933 & 2010-07-19 & 86 \\
\hline HIFI & $13 \mathrm{CO} J=5-4 \mathrm{C}$ & 1342201090 & 2010-07-21 & 679 \\
\hline HIFI & $13 \mathrm{CO} J=5-4 \mathrm{NW}$ & 1342201093 & 2010-07-21 & 353 \\
\hline HIFI & $13 \mathrm{CO} J=5-4 \mathrm{SE}$ & 1342201095 & $2010-0$ & 353 \\
\hline HIFI & $\mathrm{C} 18 \mathrm{O} J=5-4 \mathrm{C}$ & 1342201091 & $2010-0$ & 112 \\
\hline HIFI & $\mathrm{CO} J=6-5 \mathrm{C}$ & & & 82 \\
\hline HIFI & $\mathrm{CO} J=$ & 134 & 2010 & 82 \\
\hline HIFI & $\mathrm{O} J$ & 84 & 201( & 82 \\
\hline IFI & $\mathrm{CO} J$ & 11 & 2010 & 1753 \\
\hline IFI & $\mathrm{CO} J=$ & 1342 & 2010 & 94 \\
\hline IFI & $\mathrm{CO} J$ & 01715 & 2010 & 94 \\
\hline IFI & $\mathrm{CO} J$ & 01728 & $2010-$ & 92 \\
\hline IFI & 8-7 NW & 1729 & $2010-$ & 92 \\
\hline IFI & SE & & & 92 \\
\hline HIFI & $\mathrm{CO} J=9$ & 342200948 & $2010-0$ & 1896 \\
\hline HIFI & $\mathrm{CO} J=9-8 \mathrm{NW}$ & 1342200949 & $2010-0$ & 70 \\
\hline HIFI & $\mathrm{CO} J=9-8 \mathrm{SE}$ & 1342200950 & $2010-0$ & 70 \\
\hline HIFI & CO $J=10-9 \mathrm{C}$ & 01108 & $2010-0$ & 257 \\
\hline HIFI & $\mathrm{CO} J=10$ & 1109 & $2010-$ & 257 \\
\hline HIFI & SE & 1110 & $2010-0$ & 257 \\
\hline HIFI & & & 30 & 1896 \\
\hline HIFI & $\mathrm{CO} J$ & 776 & 2010 & 70 \\
\hline HIFI & SE & 134 & 201 & 70 \\
\hline HIFI & $=1-0 \mathrm{C}$ & 1342 & 2010 & 353 \\
\hline HIFI & {$[\mathrm{CI}] J=1-0 \mathrm{NW}$} & 1342201092 & 2010 & 112 \\
\hline HIFI & $1-0 \mathrm{SE}$ & 1342201094 & $2010-$ & 112 \\
\hline HIFI & $=2-1 \mathrm{C}$ & 1712 & $2010-$ & 88 \\
\hline HIFI & {$[\mathrm{CI}] J=2-1 \mathrm{NW}$} & 1342201713 & $2010-07-30$ & 88 \\
\hline HIFI & {$[\mathrm{CI}] J=2-1 \mathrm{SE}$} & 1342201716 & 2010-07-19 & 1884 \\
\hline HIFI & {$[\mathrm{CII}] \mathrm{C}$} & 1342213717 & 2011-02-04 & 8970 \\
\hline HIFI & [CII] NW & 1342201643 & $2010-07-28$ & 936 \\
\hline HIFI & [CII] SE & 1342201644 & $2010-07-28$ & 936 \\
\hline HIFI & [NII] & 1342201778 & 2010-07-31 & 833 \\
\hline SPIRE & SSPEC & 1342204037 & $2010-08-23$ & 5041 \\
\hline
\end{tabular}

key programme HEXGAL (PI: R. Güsten). Observations were carried out in fast-chopping dual-beam switch mode using a wobbler throw of $3^{\prime}$ for all observations, and are summarised in Table 1. Calibration was achieved through hot-cold absorber measurements. The data were recorded using the wide-band acousto-optical spectrometer, consisting of four units with a bandwidth of $1 \mathrm{GHz}$ each, covering the $4 \mathrm{GHz}$ IF for each polarisation with spectral resolutions of $1 \mathrm{MHz}$. Data were reduced using the HIPE and CLASS software packages. For each scan, we combined the four sub-bands in each polarisation to create a $4 \mathrm{GHz}$ spectrum. We subtracted first-order baselines. For each line, we inspected the result in each polarisation ( $\mathrm{H}$ and $\mathrm{V}$ ) separately. The continuum and line amplitudes agreed within $15 \%$. We have used the calibration in Table 5.5 of the online HIFI Observer's Manual to convert antenna temperatures to main-beam temperatures and flux densities. Between 480 and $960 \mathrm{GHz}$, the main-beam efficiency is almost constant, dropping from $\eta_{\mathrm{mb}}=0.76$ to $\eta_{\mathrm{mb}}=0.74$, and the antenna temperature to flux conversion factor likewise changes only little from 464 to $472 \mathrm{Jy} / \mathrm{K}$.

With the exception of the $\mathrm{C}^{18} \mathrm{O}(5-4)$ and [N II] lines (neither of which was detected), we observed all lines at three positions: the center, and two positions offset by $10^{\prime \prime}$ to either side in a position angle of $45^{\circ}$ in order to separately sample emission from the CND. The central position was observed with reasonably 
Table 2. ${ }^{12} \mathrm{CO}$ observations.

\begin{tabular}{|c|c|c|c|c|c|c|c|c|}
\hline \multirow[t]{2}{*}{ Transition } & \multirow{2}{*}{$\begin{array}{l}\text { Frequency } \\
\qquad(\mathrm{GHz})\end{array}$} & \multirow[t]{2}{*}{ Telescope } & \multirow{2}{*}{$\begin{array}{l}\text { Beam } \\
\text { Size } \\
\left({ }^{\prime \prime}\right)\end{array}$} & \multirow{2}{*}{$\begin{array}{l}\text { Offset } \\
\left({ }^{\prime \prime}\right)\end{array}$} & \multirow{2}{*}{$\begin{array}{c}\text { Peak } \\
T_{\mathrm{mb}} \\
(\mathrm{mK})\end{array}$} & \multicolumn{2}{|c|}{$\int T_{\mathrm{mb}} \mathrm{d} V$} & \multirow{2}{*}{$\begin{array}{c}\int S_{v} \mathrm{~d} V \\
\text { Integrated } \\
\left(\mathrm{Jy}_{\mathrm{km} \mathrm{s}}^{-1}\right)\end{array}$} \\
\hline & & & & & & $\begin{array}{r}\text { Integrated }^{a} \\
(\mathrm{Kk}\end{array}$ & $\begin{array}{l}\text { Gauss fit }{ }^{b} \\
\left.\mathrm{~s}^{-1}\right)\end{array}$ & \\
\hline \multirow[t]{5}{*}{$J=1-0$} & 115.271 & SEST & 45 & 0,0 & 357 & 58 & 76 & $1096 \pm 164$ \\
\hline & & & & $-16.4,+11.5$ & 330 & 90 & 89 & $1701 \pm 170$ \\
\hline & & & & $-32.8,+23.0$ & 440 & 86 & 86 & $1625 \pm 163$ \\
\hline & & & & $+16.4,-11.5$ & 450 & 88 & 84 & $1663 \pm 166$ \\
\hline & & & & $+32.8,-23.0$ & 440 & 86 & 89 & $1625 \pm 170$ \\
\hline \multirow[t]{5}{*}{$J=2-1$} & 230.538 & SEST & 23 & 0,0 & 520 & 84 & 104 & $1722 \pm 258$ \\
\hline & & & & $-16.4,+11.5$ & 540 & 62 & 63 & $1271 \pm 170$ \\
\hline & & & & $-32.8,+23.0$ & 500 & 61 & 61 & $1251 \pm 125$ \\
\hline & & & & $+16.4,-11.5$ & 505 & 65 & 62 & $1333 \pm 133$ \\
\hline & & & & $+32.8,-23.0$ & 440 & 62 & 59 & $1271 \pm 127$ \\
\hline \multirow[t]{7}{*}{$J=3-2$} & 345.796 & JCMT & 14 & 0,0 & 492 & 80 & 92 & $1481 \pm 222$ \\
\hline & & & & $-18.8,+9.4$ & 315 & 33 & - & $602 \pm 90$ \\
\hline & & & & $-34.5,+25.0$ & 765 & 48 & - & $875 \pm 131$ \\
\hline & & & & $-40.7,+28.1$ & 635 & 55 & - & $1057 \pm 157$ \\
\hline & & & & $+18.4,-9.4$ & 875 & 60 & - & $1094 \pm 164$ \\
\hline & & & & $+34.5,-25.0$ & 635 & 61 & - & $1167 \pm 175$ \\
\hline & & & & $+40.7,-28.1$ & 365 & 30 & - & $547 \pm 82$ \\
\hline \multirow[t]{2}{*}{$J=4-3$} & 461.041 & APEX & 14 & 0,0 & 287 & 57 & 64 & $1651 \pm 330$ \\
\hline & & SPIRE & 41 & 0,0 & & & & $7364 \pm 638$ \\
\hline \multirow{4}{*}{$J=5-4$} & 576.268 & SPIRE & 33 & 0,0 & & & & $5196 \pm 234$ \\
\hline & & HIFI & 38 & 0,0 & 75 & 13.7 & 14.6 & $4709 \pm 471$ \\
\hline & & & & $-7.5,+7.5$ & $65:$ & 12 & - & - \\
\hline & & & & $+7.5,-7.5$ & 73: & 13 & - & - \\
\hline \multirow[t]{5}{*}{$J=6-5$} & 691.473 & APEX & 9 & 0,0 & 99 & 27 & 32 & $787 \pm 187$ \\
\hline & & SPIRE & 29 & 0,0 & & & & $2768 \pm 88$ \\
\hline & & HIFI & 33 & 0,0 & 30 & 9.9 & 10.3 & $3460 \pm 573$ \\
\hline & & & & $-7.5,+7.5$ & 48: & 8: & - & \\
\hline & & & & $+7.5,-7.5$ & 48: & 8: & - & \\
\hline \multirow[t]{4}{*}{$J=7-6$} & 806.652 & SPIRE & 35 & 0,0 & & & & $1977 \pm 70$ \\
\hline & & HIFI & 27 & 0,0 & 20 & 4.4 & 4.7 & $1548 \pm 232$ \\
\hline & & & & $-7.5,+7.5$ & 35: & $2:$ & - & - \\
\hline & & & & $+7.5,-7.5$ & 43: & 5: & - & - \\
\hline \multirow[t]{4}{*}{$J=8-7$} & 921.799 & SPIRE & 36 & 0,0 & & & & $1548 \pm 102$ \\
\hline & & HIFI & 25 & 0,0 & 21 & 4.7 & 5.2 & $1669 \pm 419$ \\
\hline & & & & $-7.5,+7.5$ & 32: & 3: & - & - \\
\hline & & & & $+7.5,-7.5$ & 31: & 2: & - & - \\
\hline \multirow[t]{4}{*}{$J=9-8$} & 1036.912 & SPIRE & 18.7 & 0,0 & & & & $632 \pm 71$ \\
\hline & & HIFI & 23 & 0,0 & 14 & 2.7 & 2.9 & $1259 \pm 212$ \\
\hline & & & & $-7.5,+7.5$ & 50: & $4:$ & - & - \\
\hline & & & & $+7.5,-7.5$ & 34: & 7: & - & - \\
\hline \multirow[t]{2}{*}{$J=10-9$} & 1151.986 & SPIRE & 17.1 & 0,0 & & & & $341 \pm 62$ \\
\hline & & HIFI & 20 & average 3 pos & $<14$ & $0.5:$ & - & $281 \pm 93$ \\
\hline$J=11-10$ & 1267.014 & SPIRE & 17.6 & 0,0 & & & & $306 \pm 68$ \\
\hline$J=12-11$ & 1381.995 & SPIRE & 16.9 & 0,0 & & & & $183 \pm 57$ \\
\hline \multirow[t]{2}{*}{$J=13-12$} & 1496.923 & SPIRE & 16.8 & 0,0 & & & & $393 \pm 64$ \\
\hline & & HIFI & 15 & average 3 pos & $<40$ & $<1.2$ & - & $<435$ \\
\hline
\end{tabular}

Notes. ${ }^{(a)}$ Summation over all amplitudes in the velocity interval $300-800 \mathrm{~km} \mathrm{~s}^{-1}$, i.e. the integrated emission line intensity was not corrected for absorption. ${ }^{(b)}$ Sum of one or more Gaussians fitted to the observed line profile, excluding the velocity range between $500 \mathrm{~km} \mathrm{~s}^{-1}$ and $625 \mathrm{~km} \mathrm{~s}^{-1}$, i.e. the integrated emission line intensity is to first order unaffected by absorption.

good signal-to-noise ratios in the $J=5-4, J=7-6, J=9-8$, and $J=13-12$ transitions of ${ }^{12} \mathrm{CO}$, as were the $J=13-12$ ${ }^{12} \mathrm{CO} \mathrm{NW}$ and SE offset positions. The other CO measurements had much shorter durations resulting in poorly defined spectral profiles, but the [C I] and [C II] lines were again observed with (very) good signal-to-noise ratios at all positions. As shown in Tables 2-4, the HIFI beam sizes ranged from $38^{\prime \prime}$ at $576 \mathrm{GHz}$ to $11^{\prime \prime}$ at $1900 \mathrm{GHz}$. The profiles observed with HIFI are shown in Figs. 1 and 2.

\subsection{APEX $12 m$}

Between 2007 and 2011 we have used the Vertex Antennentechnik APEX ${ }^{2}$ 12-m telescope (Güsten et al. 2006) to observe the nucleus of NGC 5128 in the $J=3-2{ }^{13} \mathrm{CO}$ transition

2 The Atacama Pathfinder Experiment (APEX) is a collaboration between the Max-Planck-Institut für Radioastronomie (MPIfR), the European Southern Observatory (ESO), and the Onsala Space Observatory (OSO). 
Table 3. ${ }^{13} \mathrm{CO}$ and $\mathrm{HCN}$ observations.

\begin{tabular}{|c|c|c|c|c|c|c|c|c|c|c|c|}
\hline Transition & $\begin{array}{c}\text { Frequency } \\
(\mathrm{GHz})\end{array}$ & Telescope & $\begin{array}{l}\text { Beam } \\
\text { Size } \\
\left({ }^{\prime \prime}\right)\end{array}$ & $\begin{array}{l}\text { Offset } \\
\left({ }^{\prime \prime}\right)\end{array}$ & $\begin{array}{c}\text { Peak } \\
T_{\mathrm{mb}} \\
(\mathrm{mK})\end{array}$ & $\begin{array}{c}\int T_{\mathrm{mb}} \mathrm{d} V \\
\left(\mathrm{Kkm} \mathrm{s}^{-1}\right)\end{array}$ & $\begin{array}{c}\int S_{\nu} \mathrm{d} V \\
\text { Integrated } \\
\left(\mathrm{Jy} \mathrm{km} \mathrm{s}^{-1}\right)\end{array}$ & All & $\begin{array}{l}\text { Ratio } \\
\text { ETD }\end{array}$ & $\begin{array}{c}{ }^{12} \mathrm{CO} /{ }^{13} \mathrm{CO} \\
\mathrm{CND} \mathrm{SE}\end{array}$ & CND NW \\
\hline \multicolumn{12}{|c|}{${ }^{13} \mathrm{CO}$} \\
\hline$J=1-0$ & 110.201 & SEST & 47 & $\begin{array}{c}0,0 \\
-16,+12 \\
-32,+24 \\
-40,+28 \\
-48,+32 \\
+16,-12 \\
+32,-24 \\
+40,-32 \\
+48,032\end{array}$ & $\begin{array}{l}39 \\
34 \\
23 \\
19 \\
13 \\
36 \\
26 \\
19 \\
18\end{array}$ & $\begin{array}{l}7.4 \\
7.5 \\
3.7 \\
2.7 \\
1.8 \\
6.5 \\
3.8 \\
3.1 \\
2.1\end{array}$ & $\begin{array}{c}140 \pm 28 \\
142 \pm 15 \\
70 \pm 11 \\
51 \pm 8 \\
34 \pm 5 \\
123 \pm 18 \\
72 \pm 11 \\
59 \pm 9 \\
40 \pm 6\end{array}$ & $\begin{array}{c}9 \pm 2 \\
23 \\
- \\
- \\
23 \\
- \\
-\end{array}$ & $10 \pm 2$ & $10 \pm 4$ & $11 \pm 4$ \\
\hline$J=2-1$ & 220.399 & SEST & 24 & $\begin{array}{c}0,0 \\
-16.4,+11.5 \\
-32.8,+23.0 \\
-41.0,+28.5 \\
+16.4,-11.5 \\
+32.8,-23.0 \\
+41.0,-28.5 \\
+49.2,-34.2\end{array}$ & $\begin{array}{l}46 \\
38 \\
54 \\
36 \\
52 \\
50 \\
48 \\
48\end{array}$ & $\begin{array}{l}7.8 \\
5.4 \\
7.4 \\
4.2 \\
8.2 \\
7.1 \\
7.0 \\
6.6\end{array}$ & $\begin{array}{c}160 \pm 32 \\
111 \pm 17 \\
152 \pm 23 \\
86 \pm 13 \\
168 \pm 25 \\
146 \pm 22 \\
144 \pm 22 \\
135 \pm 20\end{array}$ & $\begin{array}{c}11 \pm 1 \\
12 \\
8 \\
- \\
7.5 \\
8.5 \\
- \\
-\end{array}$ & $13 \pm 2$ & $13 \pm 1.5$ & $11 \pm 1.5$ \\
\hline$J=5-4$ & $\begin{array}{r}330.588 \\
550.926\end{array}$ & $\begin{array}{l}\text { JCMT } \\
\text { APEX }\end{array}$ & $\begin{array}{l}15 \\
18\end{array}$ & $\begin{array}{c}0,0 \\
0,0 \\
-20.8,+12.0 \\
+20.8,-12.0 \\
\text { average }\end{array}$ & $\begin{array}{l}41 \\
87 \\
41 \\
24 \\
1.2\end{array}$ & $\begin{array}{l}6.2 \\
14 \\
6.3 \\
4.4 \\
0.7 \\
\end{array}$ & $\begin{array}{l}182 \pm 36 \\
419 \pm 84 \\
189 \pm 28 \\
132 \pm 20 \\
244 \pm 85\end{array}$ & $14 \pm 2$ & $14 \pm 2$ & $17 \pm 4$ & $13 \pm 2$ \\
\hline \multicolumn{12}{|c|}{ HCN } \\
\hline$J=1-0$ & 88.632 & SEST & 57 & $\begin{array}{c}0,0 \\
-16.4,+11.4 \\
-49.2,+34.2 \\
+16.4,-11.4 \\
+49.2,+34.2\end{array}$ & $\begin{array}{c}0.026 \\
0.015 \\
<0.008 \\
0.015 \\
<0.008 \\
\end{array}$ & $\begin{array}{r}1.60^{b} \\
0.59^{b} \\
0.24 \\
0.20^{b} \\
0.25 \\
\end{array}$ & $\begin{array}{l}- \\
- \\
- \\
- \\
-\end{array}$ & $\begin{array}{l}- \\
- \\
- \\
- \\
-\end{array}$ & & & \\
\hline
\end{tabular}

Notes. ${ }^{(a)}$ Ratios determined by (partial) fitting ${ }^{13} \mathrm{CO}$ profile to ${ }^{12} \mathrm{CO}$ profile; ${ }^{(b)}$ Profiles dominated by absorption; integrated values cover velocity ranges $V_{\mathrm{LSR}}=300-500 \mathrm{~km} \mathrm{~s}^{-1}$ and $V_{\mathrm{LSR}}=600-800 \mathrm{~km} \mathrm{~s}^{-1}$ only.

Table 4. $\mathrm{C}^{\circ}$ and $\mathrm{C}^{+}$observations.

\begin{tabular}{|c|c|c|c|c|c|c|c|c|}
\hline \multirow[t]{2}{*}{ Transition } & \multirow{2}{*}{$\begin{array}{c}\text { Frequency } \\
(\mathrm{GHz})\end{array}$} & \multirow[t]{2}{*}{ Telescope } & \multirow{2}{*}{$\begin{array}{c}\text { Beam } \\
\text { Size } \\
\left({ }^{\prime \prime}\right) \\
\end{array}$} & \multirow{2}{*}{$\begin{array}{l}\text { Offset } \\
\left({ }^{\prime \prime}\right)\end{array}$} & \multirow{2}{*}{$\begin{array}{c}\text { Peak } \\
T_{\mathrm{mb}} \\
(\mathrm{K}) \\
\end{array}$} & \multicolumn{2}{|c|}{$\int T_{\mathrm{mb}} \mathrm{dV}$} & \multirow{2}{*}{$\underset{\substack{\text { Integrated } \\
\left(\mathrm{Jy} \mathrm{km} \mathrm{s}^{-1}\right)}}{\int S_{\nu} \mathrm{d} V}$} \\
\hline & & & & & & $\begin{array}{r}\text { Integrated }^{a} \\
(\mathrm{~K})\end{array}$ & $\begin{array}{l}\text { Gauss fit } \\
\left.\mathrm{s}^{-1}\right)\end{array}$ & \\
\hline \multirow[t]{5}{*}[\mathrm{C}\mathrm{I}]{$J=1-0$} & 492.161 & APEX & 12.5 & 0,0 & 342 & 84.6 & 100 & $2880 \pm 432$ \\
\hline & & SPIRE & 38 & 0,0 & & & & $7014 \pm 328$ \\
\hline & & HIFI & 44.1 & 0,0 & 79 & 17.0 & 19 & $6700 \pm 1005$ \\
\hline & & & & $-7.5,+7.5$ & 86 & 18.3 & 20 & $7053 \pm 1058$ \\
\hline & & & & $+7.5,-7.5$ & 78 & 14.8 & 16 & $5642 \pm 846$ \\
\hline \multirow[t]{5}{*}[\mathrm{C}\mathrm{I}]{$J=2-1$} & 809.342 & APEX & 7.7 & 0,0 & 510 & 104.3 & 136 & $4094 \pm 614$ \\
\hline & & SPIRE & 34 & 0,0 & & & & $11196 \pm 80$ \\
\hline & & HIFI & 26.5 & 0,0 & 99 & 20.7 & 26 & $9146 \pm 1372$ \\
\hline & & & & $-7.5,+7.5$ & 81 & 18.4 & 21 & $7387 \pm 1108$ \\
\hline & & & & $+7.5,-7.5$ & 91 & 14.7 & 18 & $6332 \pm 950$ \\
\hline [N II] & 1426.1 & SPIRE & 16.9 & 0,0 & & & & $10090 \pm 97$ \\
\hline \multirow{3}{*}{ [C II] } & 1900.539 & HIFI & 11.1 & 0,0 & 885 & 140.0 & 144 & $50276 \pm 7541$ \\
\hline & & & & $-7.5,+7.5$ & 790 & 60.8 & 59 & $21228 \pm 3184$ \\
\hline & & & & $+7.5,-7.5$ & 1290 & 102.3 & 95 & $33168 \pm 4975$ \\
\hline
\end{tabular}

Notes. ${ }^{(a)}$ Summation over all amplitudes in the velocity interval $300-800 \mathrm{~km} \mathrm{~s}^{-1}$, i.e. the integrated emission line intensity was not corrected for absorption. In the case of the [CII] profiles, the velocity interval was 370 to $720 \mathrm{~km} \mathrm{~s}^{-1}$. ${ }^{(b)}$ Sum of one or more Gaussians fitted to the observed line profile, excluding the velocity range between $500 \mathrm{~km} \mathrm{~s}^{-1}$ and $625 \mathrm{~km} \mathrm{~s}^{-1}$, i.e. the integrated emission line intensity is to first order unaffected by absorption.

at $330 \mathrm{GHz}$, the $J=4-3$ and $J=6-5{ }^{12} \mathrm{CO}$ transitions at 461 and $691 \mathrm{GHz}$ respectively, and the two submillimeter [C I] transitions at 492 and $809 \mathrm{GHz}$. The location of APEX at the high elevation of $5105 \mathrm{~m}$ renders it very suitable to highfrequency observations from the ground. The observations were made with the First Light APEX Submillimeter Heterodyne 
F. P. Israel et al.: Cen A circumnuclear disk
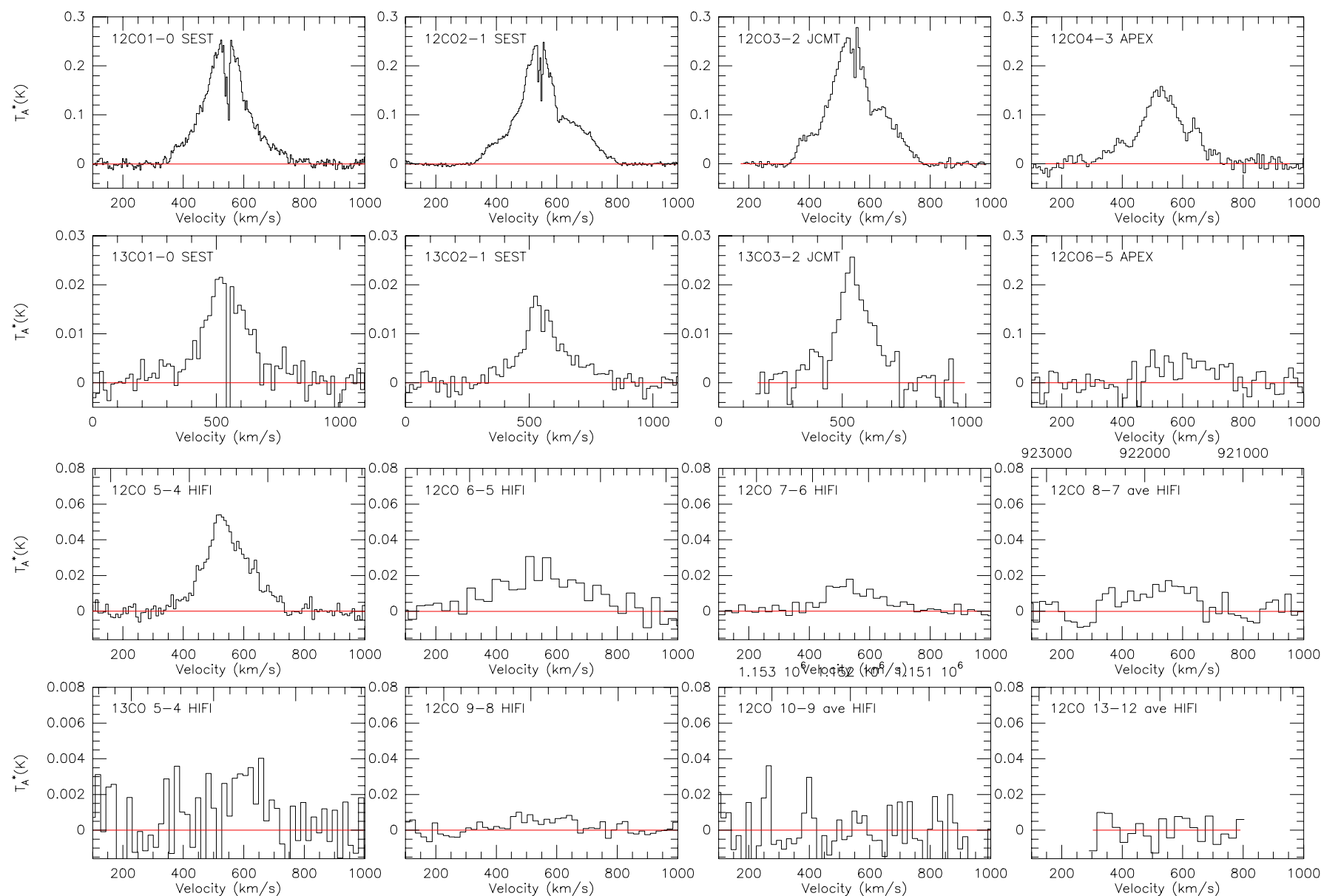

Fig. 1. Baseline-subtracted line profiles towards the center of NGC 5128 (Centaurus A) obtained with the ground-based telescopes SEST, JCMT, and APEX (top two rows) and with the HIFI instrument on-board of the Herschel Space Observatory (bottom two rows). Vertical scale is $T_{\mathrm{A}}^{*}$ in Kelvin, horizontal scale is $V_{\mathrm{LSR}}$ in $\mathrm{km} \mathrm{s}^{-1}$. Species, transition and telescope used are identified at the top left corner of each panel. The HIFI ${ }^{12} \mathrm{CO}$ results for the $J=8-7, J=10-9$, and $J=13-12$ transitions are the averages of the profile towards the nucleus and the adjacent positions separated from that by $10^{\prime \prime}$. Note that the deep absorption apparent in the ${ }^{13} \mathrm{CO}(1-0)$ profile is real; its depth to negative values reflects the weakness of the line emission relative to the (subtracted) continuum strength at the same frequency. For more details, see Sect. 2 and Tables 2 and 3 .
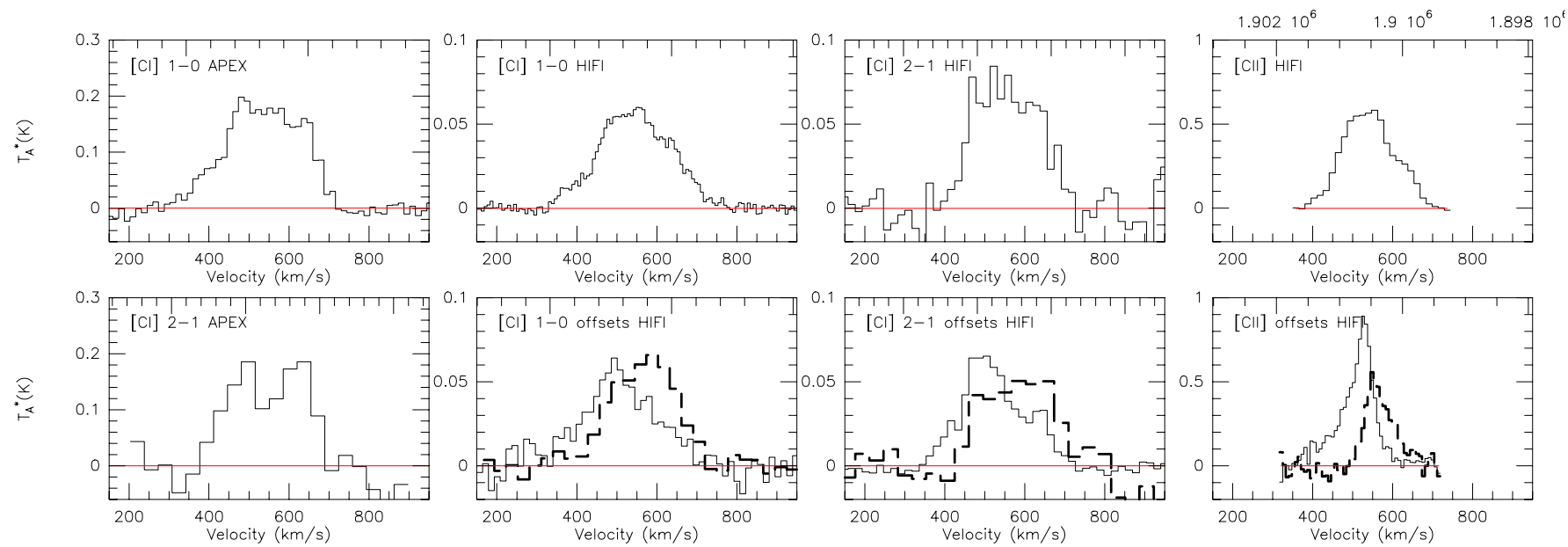

Fig. 2. Baseline-subtracted line profiles towards the center of NGC 5128 (Centaurus A) obtained with the APEX telescope and the HIFI instrument on-board the Herschel Space Observatory. The HIFI [C I] and [C II] profiles for the nuclear position, and the positions offset by $\pm 10^{\prime \prime}$ from the nucleus, are shown separately. The NW offset profile is represented by a dashed line, the SE offset profile by a solid line. Species, transition and telescope used are identified at the top left corner of each panel. Vertical scale is $T_{\mathrm{A}}^{*}$ in Kelvin, horizontal scale is $V_{\mathrm{LSR}}$ in $\mathrm{km} \mathrm{s}^{-1}$. For more details, see Table 4.

(FLASH) dual-frequency receiver (Heyminck et al. 2006) and the Carbon Heterodyne Array (CHAMP+) receiver (Güsten et al. 2008; Kasemann et al. 2006), both developed by the Max
Planck Institut für Radioastronomie in Bonn (Germany). Mainbeam efficiencies were $0.73,0.60,0.56$, and 0.43 at operating frequencies of $352,464,650$, and $812 \mathrm{GHz}$. At the same 
frequencies, the antenna temperature to flux density conversion factors were $41,48,53$, and $70 \mathrm{Jy} / \mathrm{K}$, respectively, with beam sizes ranging from $18^{\prime \prime}$ to $7.7^{\prime \prime}$.

All observations were done under excellent weather conditions with typical overall system temperatures of $2100 \mathrm{~K}$ for CHAMP+-I (SSB, $690 \mathrm{GHz}$ ), $7500 \mathrm{~K}$ for CHAMP+-II (SSB, $800 \mathrm{GHz}$ ), and $1100 \mathrm{~K}$ for FLASH-I (DSB, $460 \mathrm{GHz}$ ), $290 \mathrm{~K}$ for APEX-1 and $230 \mathrm{~K}$ for APEX-2a (both SSB). Calibration errors are estimated at 15 to $20 \%$. Observations were taken using fast Fourier transform spectrometer (FFTS; Klein et al. 2006) backends for all instruments, except CHAMP+, for which only the two central pixels were attached to the FFTS back-ends. Other CHAMP+ pixels were attached to the MPI Array Correlator System (MACS) back-ends. FFTS back-ends are able to reach resolutions of $0.12 \mathrm{MHz}\left(0.045 \mathrm{~km} \mathrm{~s}^{-1}\right.$ at $\left.800 \mathrm{GHz}\right)$, while the MACS units were used at a resolution of $1 \mathrm{MHz}\left(0.36 \mathrm{~km} \mathrm{~s}^{-1}\right.$ at $800 \mathrm{GHz}$ ). APEX absolute pointing accuracy is $\sim 2^{\prime \prime}$ (rms), but its pointing on track is accurate to $0.6^{\prime \prime}$ (rms). All observations were taken using position switching with reference positions in azimuth ranging from $600^{\prime \prime}$ to $3600^{\prime \prime}$. The results are shown in Figs. 1 and 2, and summarised in Tables 2-4.

\subsection{JCMT $15 m$}

The $15 \mathrm{~m}$ James Clerk Maxwell Telescope (JCMT) ${ }^{3}$ on top of Mauna Kea (Hawaii) was used between 2003 and 2005 to measure the $J=3-2$ transitions of ${ }^{12} \mathrm{CO}$ and ${ }^{13} \mathrm{CO}$ at 345 and $330 \mathrm{GHz}$ respectively towards the nucleus of Centaurus A. At the observing frequencies, the beam size was $14^{\prime \prime}$ and the main-beam efficiency was 0.62 . The antenna temperature to flux density conversion was $29.4 \mathrm{Jy} / \mathrm{K}$. We used the dual-polarisation receiver $\mathrm{RxB}$ and the Digital Autocorrelating Spectrometer (DAS) back-end in wide-band mode, with total band-widths of $920 \mathrm{MHz}\left(800 \mathrm{~km} \mathrm{~s}^{-1}\right)$ and $500 \mathrm{MHz}\left(435 \mathrm{~km} \mathrm{~s}^{-1}\right)$, providing velocity resolutions of 0.65 and $0.325 \mathrm{~km} \mathrm{~s}^{-1}$ respectively. As seen from Hawaii, Centaurus A culminates at an elevation of only $23^{\circ}$ above the horizon. We observed the object only when above $20^{\circ}$, i.e. about $2 \mathrm{~h}$ per day. All observations were taken in beam-switching mode with a throw of $3^{\prime}$ in azimuth. The results are shown in Fig. 1, and summarised in Tables 2 and 3.

\subsection{SEST $15 m$}

We have used the $15 \mathrm{~m}$ Swedish ESO Submillimetre Telescope ${ }^{4}$ (SEST; Booth et al. 1989) on top of Cerro La Silla (Chile) to observe the $J=1-0 \mathrm{HCN}, J=1-0$, and $J=2-1{ }^{12} \mathrm{CO}$ and ${ }^{13} \mathrm{CO}$ transitions with angular resolutions of $57 ", 45^{\prime \prime}$, and $23^{\prime \prime}$ respectively. In order to convert observed antenna temperatures to main-beam temperatures, we use efficiencies $\eta_{\mathrm{mb}}=$ $0.75,0.70$, and 0.50 respectively. Similarly, we derive flux densities from the observed antenna temperatures by applying the respective conversion factors 25,27 , and $41 \mathrm{Jy} / \mathrm{K}$. All observations were made in the double-beam switching mode, with a throw of $12^{\prime}$ and a frequency of $6 \mathrm{~Hz}$, producing excellent baseline stability. In the early period 1989-1993, we used a relatively noisy Schottky barrier diode receiver. Between 1996 and 2003

\footnotetext{
3 The James Clerk Maxwell Telescope is operated by the Joint Astronomy Centre on behalf of the Science and Technology Facilities Council of the UK, the National Research Council of Canada, and (until 31 March 2013) the Netherlands Organisation for Scientific Research.

4 The Swedish-ESO Submillimeter Telescope was operated jointly by the European Southern Observatory (ESO) and the Swedish Science Research Council (NFR).
}

we obtained spectra with the more sensitive SIS receiver, using high- and low-resolution AOS back-ends in parallel. The data presented in this paper were taken with the latter, which had a total bandwidth of $500 \mathrm{MHz}$ (later $1 \mathrm{GHz}$ ), and a resolution of $1 \mathrm{MHz}$ (later $1.4 \mathrm{MHz}$ ). The results are shown in Fig. 1, and summarised in Tables 2 and 3.

\section{Results}

\subsection{Complex nature of the observed nuclear line profiles}

The complexity of the individual line profiles is particularly obvious in the lower $J$ transitions of ${ }^{12} \mathrm{CO}$ (Fig. 1). These illustrate the presence of the various contributions that are due to physically distinct components in the line of sight to the nucleus (see Israel 1992, 1998). These are: (a) very broad line emission at $300 \mathrm{~km} \mathrm{~s}^{-1}<V_{\mathrm{LSR}}<800 \mathrm{~km} \mathrm{~s}^{-1}$ sampling the rapidly rotating compact CND, fully covered by beams larger than $20^{\prime \prime}$, but progressively more resolved in smaller beams; (b) narrower line emission roughly at $450 \mathrm{~km} \mathrm{~s}^{-1}<V_{\mathrm{LSR}}<650 \mathrm{~km} \mathrm{~s}^{-1}$, most prominent in the lower ${ }^{12} \mathrm{CO}$ transitions, that samples the much larger and fully resolved ETD, and (c) a pattern of absorption against the nuclear continuum point source (but not the extended dust continuum) in the range $500 \mathrm{~km} \mathrm{~s}^{-1}<V_{\mathrm{LSR}}<625 \mathrm{~km} \mathrm{~s}^{-1}$. Because the continuum emission from the nucleus is completely unresolved even in the smallest observing beams, the absorption features sample the molecular ISM in both the ETD and CND along the line of sight to the nucleus in a very narrow pencilbeam, less than $0.1^{\prime \prime}$ across (i.e. $<2 \mathrm{pc}$ ).

In the remainder of this paper, we will first briefly discuss the continuum emission, and then concentrate on the line emission, i.e. contributions (a) and (b). Analysis and discussion of the line absorption (contribution (c)), based on profiles with higher spectral resolution than shown here, will be presented in a subsequent paper.

\subsection{Nuclear continuum emission}

In the beams used to obtain the velocity-resolved profiles, the (variable) flux of the nuclear point source is dominant, and dust continuum emission is negligible (cf. Israel et al. 2008). However, at the higher frequencies shown in Fig. 3, there is a steadily increasing contribution by thermal emission from dust extended over the beam area. The unresolved nuclear continuum spectrum can be approximated by a power-law $F_{v} \propto v^{-0.36}$ (Meisenheimer et al. 2007). From APEX-1 and APEX-2a observations of the continuum underlying the $\mathrm{HCO}^{+}$line emission at $267 \mathrm{GHz}$ and $355 \mathrm{GHz}$ made in June, 2010 (i.e. close to the SPIRE observing date of August, 2010) we extrapolate a flux density $F_{461 \mathrm{GHz}}=8.2 \pm 0.3 \mathrm{Jy}$. As this is indistinguishable from the value implied by Fig. 3, we conclude that at the lowest frequencies observed with SPIRE the continuum flux is still wholly due to the nucleus. At the highest observed SPIRE frequencies around $1500 \mathrm{GHz}$, the nuclear flux has decreased to $F_{1500 \mathrm{GHz}} \approx 5 \mathrm{Jy}$, and contributes no more than $15 \%$ to the continuum flux measured in the aperture.

\subsection{Spectral line flux distributions}

\subsubsection{Integral CND/ETD line profiles}

First, we will analyse the observed (CND/ETD) spectral line emission without attempting to separate it into the individual ETD and CND contributions. This allows us to use well-defined 

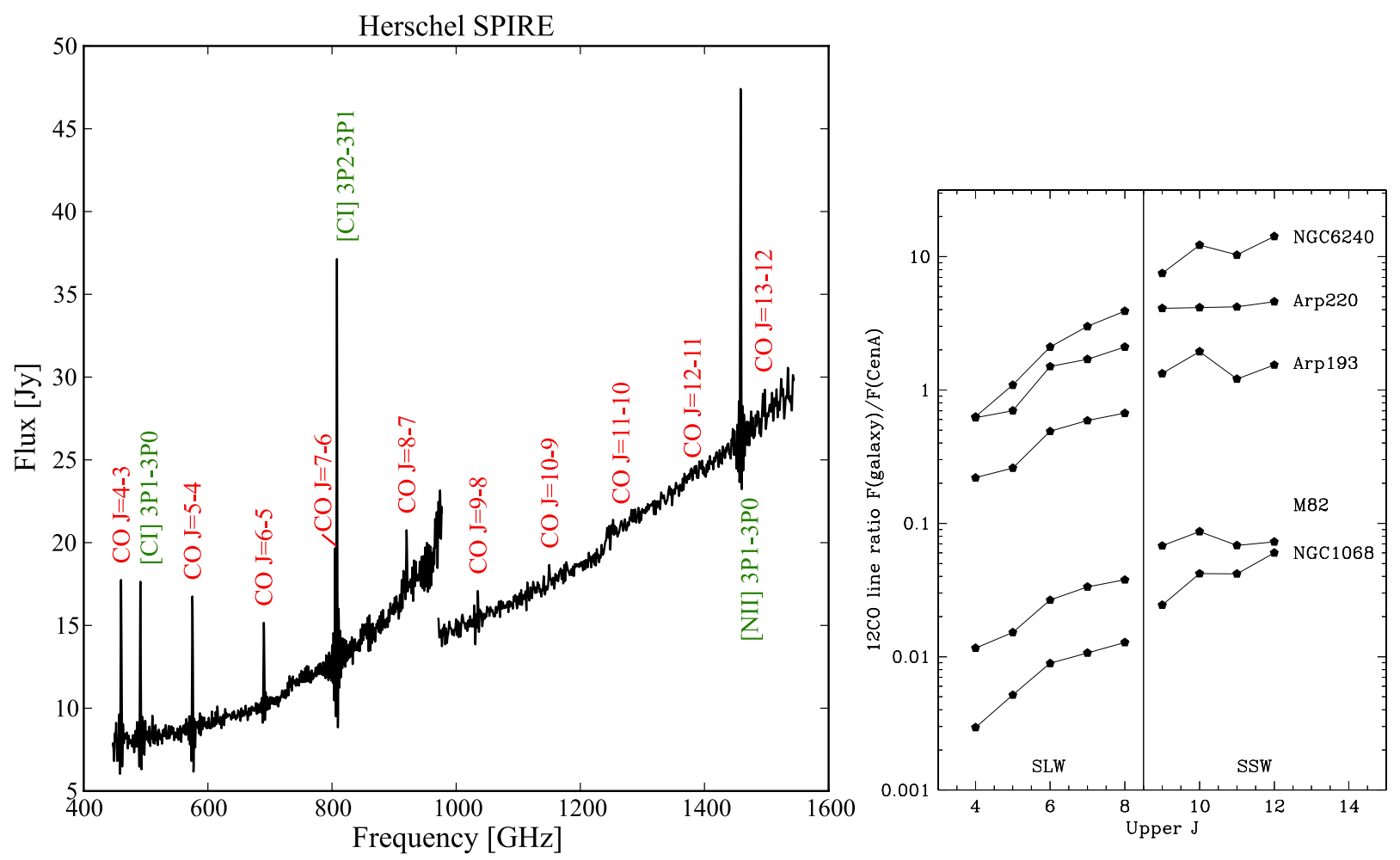

Fig. 3. Left: full submillimeter spectrum of the center of NGC 5128 (Centaurus A) obtained with the SPIRE instrument on board the Herschel Space Observatory (See Sect. 2). Species and transitions are identified throughout. The jump in the continuum at $944 \mathrm{GHz}$ is caused by the different angular resolutions of the SLW and the SSW (see Sect. 2). The SPIRE continuum contains a contribution from the point source nucleus of about $8.2 \mathrm{Jy}$ at $460 \mathrm{GHz}$, slowly decreasing with frequency. The remaining continuum is due to extended thermal emission from dust, increasing with frequency. Vertical scale is flux in Jansky, horizontal scale is frequency in GHz. For more details, see Tables 2 and 3. Right: comparison of SPIRE CO line fluxes of the NGC 5128 center with those of the starburst galaxy M 82, the AGN+starburst NGC 1068, and the (U)LIRGs Arp 193, Arp 220, and NGC 6240. Mrk 231 has not been marked separately as its spectral ladder is identical to that of NGC 6240. The ratio of the galaxy CO line flux to the Cen A CO line flux is shown for each $J$ transition, as observed by SPIRE without correction for finite beam-size, source extent, and beam efficiency. The vertical line separates measurements obtained with the SSW from those obtained with the SLW in a beam roughly twice as wide.

integrated line intensities (such as those from SPIRE) up to the $J=12-11{ }^{12} \mathrm{CO}$ transition, not hampered by the uncertainties inherent in component decomposition, and suitable for comparison with measurements of other galaxy centers. This is particularly important for the highest observed line transitions, which suffer from increasingly poor baseline-definition and signal-tonoise ratios in the velocity-resolved HIFI measurements. The measured emission line intensities, and the different beam sizes used, are given in Tables 2-4.

In constructing the NGC $5128 \mathrm{CO}$ spectral line ladder we need to take into account the variation in beam sizes. The CND and the ETD have finite but different extent, and the observed emission line fluxes must be normalised to the same beamsize. To accomplish this, we have taken the ALMA Band 6 high-resolution mosaic of the $J=2-1{ }^{12} \mathrm{CO}$ emission from Centaurus A obtained in the public ALMA Calibration and Science Verification program. From these data, we have constructed a series of maps at spatial resolutions identical to those of our single-dish line observations. From each of these maps, we have extracted the central (nuclear) line profile and determined its integral value. Assuming that the distribution on the sky of CO emitting clouds is identical for all transitions, we have determined the beam normalisation factors (BNFs) required to relate the emission line fluxes to one another. These factors are included in Table 5.

When more than one independent measurement was available, we took the error-weighted mean to derive the flux. In all these cases, the derived normalised fluxes agree reasonably well with one another, and also with values obtained from linear interpolation between measurements with beams larger respectively smaller than $22^{\prime \prime}$. For instance, our normalised flux for the [C II] line is only $10 \%$ above the value interpolated from the HIFI (11" beam) and ISO-LWS (70" beam) measurements published by Unger et al. (2000). This is quite gratifying, as the large beam normalisation factors appropriate to such small beams are at the limit of reliability.

\subsubsection{Absorption correction and normalisation}

Since the absorption occurs against the continuum emission from a nucleus very much smaller than any of the beams used, the absorption line intensity, unlike the emission line intensity, is independent of beam size. We have determined the magnitude of the absorption by subtracting the integrated line fluxes from the Gaussian-fitted line fluxes. This amount is once again added to the normalised emission line flux to finally yield the normalised and corrected line luminosities listed in Table 5. We have adopted an uncertainty in the absorption line flux of $50 \%$.

Because of the poor definition of the line profiles beyond the $J=7-6$ transition, we have not attempted to correct for absorption losses at the higher ${ }^{12} \mathrm{CO}$ transitions. We believe that the effect of this on the analysis is limited, as Table 5 shows that the magnitude of the flux absorbed against the nuclear continuum decreases steadily from $J=2-1{ }^{12} \mathrm{CO}$ upwards. This is probably due to decreasing absorption optical depths at increasing 
Table 5. Normalized spectral line emission distribution.

\begin{tabular}{|c|c|c|c|c|c|}
\hline Transition & Obs. & \multicolumn{2}{|c|}{$\left(\mathrm{Jy} \mathrm{km} \mathrm{s}^{-1}\right)$} & $F_{\text {abs }}{ }^{c}$ & $\begin{array}{c}L_{\text {cor }}{ }^{d} \\
\left(10^{4} L_{\odot}\right)\end{array}$ \\
\hline \multicolumn{6}{|c|}{ The ${ }^{12} \mathrm{CO}$ ladder } \\
\hline$J=1-0$ & SEST & 0.53 & $583 \pm 87$ & 340 & $0.16 \pm 0.05$ \\
\hline$J=2-1$ & SEST & 0.99 & $1705 \pm 256$ & 410 & $0.72 \pm 0.20$ \\
\hline$J=3-2$ & JCMT & 2.13 & $3151 \pm 473$ & 220 & $1.71 \pm 0.29$ \\
\hline \multirow[t]{2}{*}{$J=4-3$} & APEX & 2.13 & $3513 \pm 703$ & 195 & $2.83 \pm 0.61$ \\
\hline & SPIRE & 0.57 & $4208 \pm 365$ & & \\
\hline \multirow[t]{2}{*}{$J=5-4$} & SPIRE & 0.66 & $3441 \pm 155$ & 170 & $2.87 \pm 0.20$ \\
\hline & HIFI & 0.60 & $2803 \pm 280$ & & \\
\hline \multirow[t]{2}{*}{$J=6-5$} & SPIRE & 0.75 & $2066 \pm 66$ & 145 & $2.30 \pm 0.15$ \\
\hline & HIFI & 0.66 & $2291 \pm 380$ & & \\
\hline \multirow[t]{2}{*}{$J=7-6$} & SPIRE & 0.63 & $1267 \pm 46$ & 140 & $1.58 \pm 0.13$ \\
\hline & HIFI & 0.79 & $1229 \pm 185$ & & \\
\hline \multirow[t]{2}{*}{$J=8-7$} & SPIRE & 0.62 & $961 \pm 63$ & & $1.48 \pm 0.20$ \\
\hline & HIFI & 0.89 & $1477 \pm 370$ & & \\
\hline \multirow[t]{2}{*}{$J=9-8$} & SPIRE & 1.25 & $790 \pm 89$ & & $1.44 \pm 0.16$ \\
\hline & HIFI & 0.99 & $1246 \pm 210$ & & \\
\hline \multirow[t]{2}{*}{$J=10-9$} & SPIRE & 1.45 & $494 \pm 90$ & & $0.76 \pm 0.15$ \\
\hline & HIFI & 1.14 & $319 \pm 106$ & & \\
\hline$J=11-10$ & SPIRE & 1.39 & $425 \pm 120$ & & $0.79 \pm 0.22$ \\
\hline$J=12-11$ & SPIRE & 1.47 & $269 \pm 90$ & & $0.55 \pm 0.18$ \\
\hline \multicolumn{6}{|c|}{ The ${ }^{13} \mathrm{CO}$ ladder } \\
\hline \multirow{5}{*}{$\begin{array}{l}J=1-0 \\
J=2-1 \\
J=3-2 \\
J=4-3 \\
J=5-4\end{array}$} & & & & & $0.017 \pm 0.007$ \\
\hline & & & & & $0.065 \pm 0.019$ \\
\hline & & & & & $0.122 \pm 0.027$ \\
\hline & & & & & - \\
\hline & & & & & $0.16 \pm 0.0 .04$ \\
\hline \multicolumn{6}{|c|}{ The carbon $\mathrm{C}^{\circ}$ and $\mathrm{C}^{+}$lines } \\
\hline \multirow[t]{3}{*}[\mathrm{C}\mathrm{I}]{$(1-0)$} & APEX & 2.17 & $6149 \pm 922$ & 655 & $4.2 \pm 0.8$ \\
\hline & SPIRE & 0.63 & $4418 \pm 207$ & & \\
\hline & HIFI & 0.58 & $5360 \pm 804$ & & \\
\hline \multirow[t]{3}{*}[\mathrm{C}\mathrm{I}]{$(2-1)$} & APEX & 7: & - & 1782 & $11.1 \pm 2.4$ \\
\hline & SPIRE & 0.68 & $7613 \pm 761$ & & \\
\hline & HIFI & 0.81 & $7408 \pm 1111$ & & \\
\hline [C II] & HIFI & 2.8 & $140773 \pm 21115$ & 1436 & $397 \pm 60$ \\
\hline$[\mathrm{N} \mathrm{II}]$ & SPIRE & 1.47 & $14832 \pm 2000$ & - & $31 \pm 5$ \\
\hline
\end{tabular}

Notes. ${ }^{(a)}$ Beam normalisation factor (BNF) to reduce observed emission line fluxes from Tables 2, 3, 4 to those enclosed by a 22" beam; for line fluxes expressed in $\mathrm{K} \mathrm{km} \mathrm{s}^{-1}$, multiply BNF by $\left(\theta_{\mathrm{b}} / 22\right)^{2}$, where $\theta_{\mathrm{b}}$ is the observing FWHM beam-size from Tables 2 and $4 .{ }^{(b)}$ Emission line fluxes normalised to the response of a $22^{\prime \prime}$ beam by application of the BNF. ${ }^{(c)}$ Beam-independent absorption line flux derived from the estimated difference between integrated and Gaussian-fitted line intensities in Table 2; adopted error: $50 \%$. Note that this is the total amount of absorption over the whole profile, not just the prominently visible deep absorption at systemic velocities. ${ }^{(d)}$ Final adopted emission line luminosity in a $22^{\prime \prime}$ beam, corrected for absorption line losses. For ${ }^{13} \mathrm{CO}$ we did not determine line fluxes, but derived corrected luminosities directly from the more accurate ${ }^{12} \mathrm{CO}$ values using the mean of the ${ }^{12} \mathrm{CO} /{ }^{13} \mathrm{CO}$ ratios in Table 3 .

$J$ levels, and to the slow flux decrease of the nuclear continuum itself.

For the ${ }^{13} \mathrm{CO}$ transitions we only give the normalised and corrected luminosities. Because of the relatively low signalto-noise ratios of these weaker lines, we have not attempted to process the individual profiles. Instead, we have divided the ${ }^{12} \mathrm{CO}$ luminosities by the ${ }^{12} \mathrm{CO} /{ }^{13} \mathrm{CO}$ ratios presented in Table 3. This is more accurate because these were obtained by fitting, in each transition, the ${ }^{13} \mathrm{CO}$ profiles to the much more accurate ${ }^{12} \mathrm{CO}$ profiles.

\section{Analysis and discussion}

\subsection{Comparison of the central CO ladders in different galaxies}

CO line fluxes extracted from SPIRE spectra have been published for a limited number of active (mostly Seyfert) galaxies (Pereira-Santaella 2013) and also for the relatively nearby NGC 1068 (Spinoglio et al. 2012). SPIRE data are also available for star-burst galaxies including the nearby M 82 (Panuzzo et al. 2010) as well as the more distant and much more energetic Markarian 231 (Van der Werf et al. 2010), Arp 220 (Rangwala et al. 2011), NGC 6240 (Meijerink et al. 2013), Arp 193 (Papadopoulos et al. 2013). The latter are all (ultra)luminous infrared galaxies, or (U)LIRGs. Due to their much greater distances $(D=42-107 \mathrm{Mpc})$ they were covered in their entirety, whereas the SPIRE aperture sampled only the central region in the nearby galaxies. Because $M 82$ is at the same distance as Centaurus A, the Herschel measurements with SPIRE (Panuzzo et al. 2010) and HIFI (Loenen et al. 2010) are directly comparable to those presented here. Unlike NGC 5128, M 82 has a star-burst center lacking an easily identifiable nucleus. In NGC 1068, the SPIRE-SLW aperture covers both the Seyfert AGN and the star-burst, but the SSW aperture covers only the AGN and its surroundings (cf. Fig. 2 in Spinoglio et al. 2012). It is of interest to compare the SPIRE line spectra of NGC 5128 and these galaxies and identify any differences.

The right-hand diagram in Fig. 3 shows, for each CO transition, the observed line flux ratio of the luminous star-burst galaxies and the NGC 5128 center. No errors are indicated, as the formal extraction errors are very small so that the uncertainties are dominated by systematic effects. All the low-frequency (SLW) flux ratios are seen to increase with transition. However, at the highest (SSW) frequencies, the flux ratios with Arp 220, Arp 193 and the M 82 center are constant, whereas ratios for NGC 6240 (and the ULIRG Markarian 231 which has an identical CO ladder - see Van der Werf et al. 2010; Meijerink et al. 2012) keep increasing. This pattern suggests that the most-highly excited gas fraction in NGC 5128, emitting in the highest (SSW) transitions, is similar in nature to the corresponding gas phase in the star-burst galaxies, whereas the bulk of the molecular gas in NGC 5128 (emitting in the lower transitions of the SLW region) is much less highly excited than most of the gas in the star-burst galaxies.

The NGC $5128 \mathrm{CO}$ ladder peaks in the $J=5-4{ }^{12} \mathrm{CO}$ transition (corresponding to an upper energy level temperature $T=$ $E_{\mathrm{U}} / k=83 \mathrm{~K}$ ), and then decreases to low levels barely reaching a quarter of the peak luminosity in the $J=10-9$ transition (see, for instance, Fig. 5, and also Table 6). The $\mathrm{CO}$ emission from all bright-star-burst galaxy peaks in the $J=7-6(155 \mathrm{~K})$ or $J=$ $8-7(200 \mathrm{~K})$ transitions. These galaxies have $\mathrm{CO}$ ladders that are relatively flat between the $J=5-4$ and the $J=13-12(500 \mathrm{~K})$ transitions. Their SPIRE FTS spectra show strong CO lines up to the highest observed frequencies near $1550 \mathrm{GHz}(J=13-12)$, whereas the spectrum of NGC 5128 exhibits CO lines drowning in the noise beyond $J=9-8$ near $1000 \mathrm{GHz}$. In M 82, resembling the (U)LIRGs but more modestly excited, the CO ladder peaks in the $J=7-6$ transition, and CO line luminosities decline more rapidly than in the (U)LIRGs but not nearly as fast as in NGC 5128. For instance, the $J=10-9$ transition in the M 82 center still has $60 \%$ of the luminosity in the peak transition, versus $26 \%$ for the NGC 5128 center. The comparison with the resolved AGN-galaxies is more difficult to make, but except for NGC 7582 their CO ladders peak in the $J=4-3 / J=5-4$ transitions, only slightly below the Cen A peak. All AGN ladders drop 
Table 6. Mean C/CO line luminosity ratios.

\begin{tabular}{lcccccc}
\hline \hline Ratio & \multirow{2}{*}{ NGC 5128 $^{a}$} & AGNs $^{b}$ & Star-bursts $^{c}$ & Nearby $^{d}$ & \multicolumn{2}{c}{ Milky Way $^{\text {Center }}$} \\
& & & & & Clouds $^{f}$ \\
\hline $\mathrm{CO}(10-9) / \mathrm{CO}(5-4)$ & $0.26 \pm 0.05$ & $0.38 \pm 0.08$ & $1.25 \pm 0.15$ & - & - & - \\
{$[\mathrm{C} \mathrm{I}](2-1) /[\mathrm{C} \mathrm{I}](1-0)$} & $2.6 \pm 0.5$ & $1.6 \pm 0.3$ & $3.2 \pm 0.3$ & - & $1.0 \pm 0.1$ & $0.5-1.9$ \\
{$[\mathrm{C} \mathrm{I}](1-0) / \mathrm{CO}(4-3)$} & $1.2 \pm 0.1$ & $0.6 \pm 0.1$ & $0.4 \pm 0.1$ & $0.6 \pm 0.2$ & $0.6 \pm 0.1$ & $0.05-0.45$ \\
{$[\mathrm{C} \mathrm{I}](2-1) / \mathrm{CO}(7-6)$} & $5.7 \pm 0.8$ & $1.6 \pm 0.2$ & $0.7 \pm 0.1$ & - & $1.1 \pm 0.2$ & $0.3 \pm 0.1$ \\
\hline
\end{tabular}

Notes. ${ }^{(a)}$ SPIRE data from this paper, ${ }^{(b)}$ SPIRE data from Spinoglio et al. (2012); Pereira-Santaella et al. (2013). ${ }^{(c)}$ SPIRE data from Panuzzo et al. (2010); Van der Werf et al. (2010); Rangwala et al. (2011); Meijerink et al. (2013); Papadopoulos et al. (2013); Rosenberg et al. (2013). ${ }^{(d)}$ Ground-based data from Israel \& Baas (2002); Hitschfeld et al. (2008). Note that this sample overlaps with both the SPIRE star-burst and SPIRE AGN samples. ${ }^{(e)}$ COBE data from Fixsen et al. (1999). ${ }^{(f)}$ From data compiled by Kramer et al. $(2004,2008)$.

significantly with increasing transition. This behaviour is illustrated by the $\mathrm{CO}(10-9)$ to $\mathrm{CO}(5-4)$ luminosity ratios summarized in Table 6 . The lowest ratio (corresponding to the steepest CO ladder high frequency drop) is presented by the NGC 5128 center, followed by the average AGN; the average star-burst galaxy shows a rise instead. Thus, the central NGC 5128 CO ladder is the "coolest" of all galaxies considered.

\subsection{Remarkably strong $[\mathrm{Cl}]$ emission lines}

NGC 5128 has a $J=2-1 / J=1-0[\mathrm{CI}]$ luminosity ratio of 2.6 (corresponding to a line brightness temperature ratio of 0.6 ), indistinguishable from both M 82 and NGC 1068, and more generally in-between the mean values found for the AGN centers and the star-burst disks (cf. Table 6).

However, both [C I] lines in the center of NGC 5128 are rather bright with respect to the $\mathrm{CO}$ lines, unlike the situation in the other galaxies (Table 6). In NGC 5128, the $809 \mathrm{GHz}$ $[\mathrm{CI}](2-1)$ line is almost six times stronger than its $807 \mathrm{GHz}$ $\mathrm{CO}(7-6)$ neighbour. This very high NGC 5128 [CI]/CO(7-6) ratio is unparalleled. The star-burst galaxies show the weakest [CI] lines; the AGNs have double their relative [CI] line intensity but this still falls far short of the NGC 5128 value. The $492 \mathrm{GHz}$ [CI] line is also more intense than the nearby $461 \mathrm{GHz} \mathrm{CO}(4-3)$ line. Corresponding $[\mathrm{CI}] / \mathrm{CO}(4-3)$ line ratios are much lower in the luminous star-burst galaxies. They are also lower (but not as much) in the AGNs, the Milky Way center, and in the 15 nearby galaxies of various type observed from the ground by Israel \& Baas (2002), and Hitschfeld et al. (2008). Among these, high ratios as in NGC 5128 are found only in NGC 3079, NGC 4826, NGC 4945, M 51, and Circinus. At least three of the latter galaxies have a nuclear outflow. Such high ratios provide a strong hint that an excitation mechanism other than PDR is required (Israel 2005), as does the very high $[\mathrm{CI}] /{ }^{13} \mathrm{CO}(2-1)$ ratio in NGC 5128. Indeed, the observed [CI] and CO line intensities are inconsistent with e.g. the PDR models by Kaufman et al. (1999). In these models, the observed [CI]492 GHz line intensity requires a density $n_{0} \approx 3000 \mathrm{~cm}^{-3}$, and a very high radiation field $G \geq 5 \times 10^{6} G_{0}$, whereas the intersection of the $[\mathrm{CI}] 809 \mathrm{GHz} /[\mathrm{CI}] 492 \mathrm{GHz}$ and $[\mathrm{CI}] 492 \mathrm{GHz} / \mathrm{CO}(1-0)$ line intensity ratios define a density $n_{\mathrm{o}} \approx 1000 \mathrm{~cm}^{-3}$ and a very low radiation field $G \approx 10 G_{0}$.

Finally, by comparing the intensities of a particular line obtained with different apertures, we may obtain an estimate for the size of the area emitting in that line. A very compact source will produce the same brightness in all apertures independent of size, whereas a very extended source will rapidly become brighter with increasing aperture size. We conclude from the $\mathrm{CO} J=4-3$, CO $J=7-6$, and [CI] $J=1-0$ data in Tables 4 and 2 that the $[\mathrm{CI}]$ emission arises from a much more compact region than the $J=4-3{ }^{12} \mathrm{CO}$ emission but that the sizes of the regions emitting in the $J=7-6{ }^{12} \mathrm{CO}$ and $[\mathrm{CI}]$ transitions are not very different (implying that the $J=7-6{ }^{12} \mathrm{CO}$ is also much more compact than the $J=4-3{ }^{12} \mathrm{CO}$ distribution).

Thus, the ladder of $\mathrm{CO}$ intensities from the (AGNdominated) central region of NGC 5128 is quite distinct from the CO ladders representing extragalactic star-burst environments. In NGC 5128, the CO ladder intensities decrease more rapidly and most of the molecular gas is distinctly less excited than in the other galaxies. Most remarkably, NGC 5128 has [CI] line emission (much) stronger than that of the nearest $\mathrm{CO}$ lines. This is quite unlike other galaxies, whether normal or in possession of an AGN or a star-burst, where the [CI] lines are less prominent.

\subsection{Determination of individual CND and ETD CO ladders}

Notwithstanding the advantages of analysing the combined ETD and CND line profiles as observed, the fact remains that the ETD and CND are different features, with potentially different physical properties. Unfortunately, a unique decomposition of the observed profiles is not straightforward, especially in case of the high $J$ transitions observed with Herschel-HIFI which are relatively noisy, and lack well-defined baselines. Yet, such a decomposition is required if we are to analyse the properties of the CND separately from its surroundings.

To gain a perspective on the CND, we have collected in Fig. 4 position-velocity (pV) maps of the inner 100" of NGC 5128 in the lower ${ }^{12} \mathrm{CO}$ and ${ }^{13} \mathrm{CO}$ transitions and in the $\mathrm{HCN} J=$ $1-0$ transition, in position angle $\mathrm{PA}=125^{\circ}$ anti-clockwise from north. This is along the heart line of the ETD; the more compact $\mathrm{CND}$ has $\mathrm{PA} \approx 145^{\circ}$. Besides the data shown above, we have used $J=1-0{ }^{13} \mathrm{CO}$ data from Wild et al. (1997) and $J=3-2$ ${ }^{12} \mathrm{CO}$ data from Liszt (2001). The ETD is the diagonal feature dominating the ${ }^{12} \mathrm{CO}$ maps, and the CND is the almost horizontal feature in the map center that is most obvious in the HCN map. The absorption lines close to the systemic velocity are (almost) saturated, and most prominent in the lower-frequency $J=1-0 \mathrm{HCN},{ }^{12} \mathrm{CO}$, and ${ }^{13} \mathrm{CO}$ maps. Map line intensities are in temperature units, in which the (subtracted) continuum emission and the associated absorption drops with increasing frequency.

The difference between the HCN map on the one hand, and the ${ }^{12} \mathrm{CO}$ and ${ }^{13} \mathrm{CO}$ maps on the other hand is striking. The ETD signature (diagonal feature) is very clear in ${ }^{12} \mathrm{CO}$ and ${ }^{13} \mathrm{CO}$, but absent in HCN where only the CND signature (horizontal feature) is very clear. Towards the ETD. HCN intensities are at least 

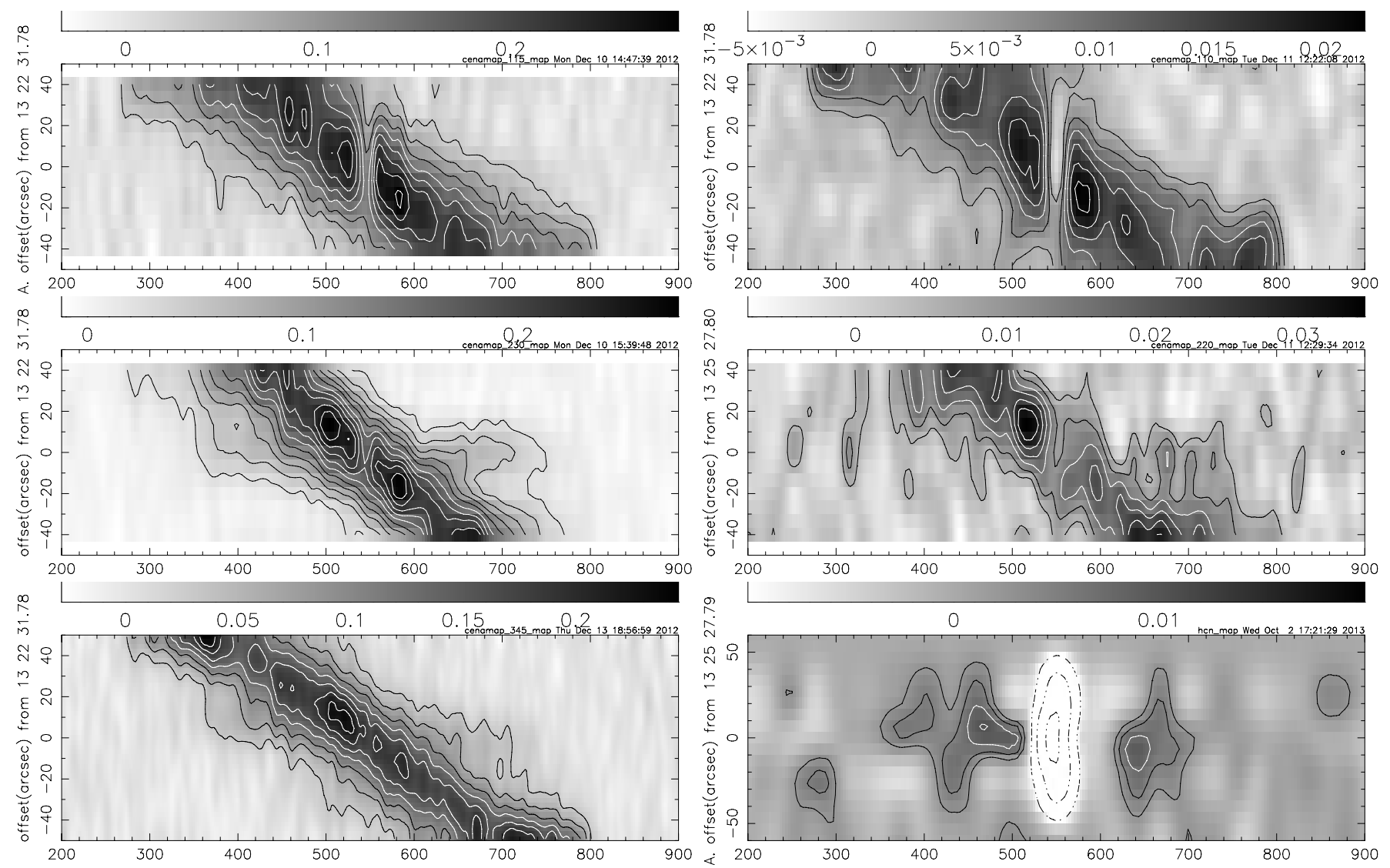

Fig. 4. Position-velocity maps of molecular line emission from the central region of Centaurus A, in position angle $125^{\circ}$ counter-clockwise from north (data summarised in Tables 2 and 3). Horizontal scales are velocity $V(L S R)$ in $\mathrm{km} \mathrm{s}^{-1}$, vertical scales are offsets from the nucleus in arcsec. The panels on the left show emission from ${ }^{12} \mathrm{CO}$; the $J=1-0, J=2-1$, and $J=3-2$ transitions are shown from top to bottom, respectively. The panels on the right show emission from ${ }^{13} \mathrm{CO}(J=1-0$ at the top, $J=2-1$ in the middle). The $J=1-0 \mathrm{HCN}$ transition is at bottom right. The $J=1-0$ maps have resolutions of $45^{\prime \prime}-55^{\prime \prime}$, all other maps have an effective resolution of $23^{\prime \prime}$. In all three ${ }^{12} \mathrm{CO}$ maps, the contours are at multiples of $50 \mathrm{mK}$ in main-beam brightness temperature. In the ${ }^{13} \mathrm{CO}$ maps contours are at multiples of $5 \mathrm{mK}(J=1-0)$ and $10 \mathrm{mK}(J=2-1)$. The HCN map contours are at multiples of $5 \mathrm{mK}$. Strong absorption is clear in all panels near $V_{\mathrm{LSR}}=550 \mathrm{~km} \mathrm{~s}^{-1}$. Emission from the rapidly rotating compact nuclear disk becomes progressively more clear with increasing $J$-transition in the ${ }^{12} \mathrm{CO}$ panels, and in the HCN map.

six times weaker than towards the CND. This factor is a lower limit because of the strong absorption towards the nucleus affecting the measured line intensity. Because the critical density for excitation of the HCN $J=1-0$ line is of the order of $10^{6} \mathrm{~cm}^{-3}$, the HCN map implies that essentially all dense gas in the line of sight towards the Cen A nucleus is actually concentrated in the CND.

The high $\mathrm{CO}$ transitions, from $J=6-5$ onwards, predominantly trace this same dense molecular gas. The CO $J=$ 7-6 transition, for instance, has a critical density $n_{\text {crit }}=4 \times$ $10^{5} \mathrm{~cm}^{-3}$. This is comparable to that of the HCN $J=1-0$ emitting gas that we have shown to be limited to the CND. Indeed, the line profiles of the higher $J$ CO transitions in Fig. 1 clearly show the signature of the rapidly rotating CND, and provide little or no evidence for a (narrow-line) contribution from the ETD. We have also noted in the preceding section that the $J=7-6 \mathrm{CO}$ emission is much more compact than the $J=4-3 \mathrm{CO}$ emission. We are therefore quite confident that from the $J=7-6$ transition onwards, the observed CO line emission is predominantly due to the CND.

The same conclusion cannot be drawn for the lower CO transitions from $J=1-0$ to $J=5-4$, where the complex nature of the velocity-resolved profiles in Fig. 1 clearly implies significant contributions from both the CND (broad plateau) and the ETD (narrower peak). We have estimated the relative contributions as follows.
Analysis of the maps in Fig. 4 shows that the profile widths from the $\mathrm{CO}$ in the ETD result from a contribution caused by a change of rotation velocity across the beam $\Delta V_{\text {rot }}=2.9 \pm$ $0.3 \mathrm{~km} \mathrm{~s}^{-1} / \operatorname{arcsec}\left(156 \pm 16 \mathrm{~km} \mathrm{~s}^{-1} / \mathrm{kpc}\right)$ and an intrinsic contribution with a velocity FWHM of $92 \pm 3 \mathrm{~km} \mathrm{~s}^{-1}$ which is independent of the observing beam. This beam-independent contribution implies the existence of a significant line-of-sight velocity dispersion $\left\langle v_{r}\right\rangle=39 \pm 1 \mathrm{~km} \mathrm{~s}^{-1}$ in the ETD.

This result allowed us to decompose the line profiles shown in Fig 1 into Gaussian components corresponding to the maximum and the minimum CND contribution respectively. In both procedures the ETD contribution was represented by a Gaussian with a fixed velocity half-width corresponding to the relevant beam-size, a central velocity allowed to deviate from the systemic velocity $\left(V_{\mathrm{LSR}}=540 \mathrm{~km} \mathrm{~s}^{-1}\right)$ by at most $10 \mathrm{~km} \mathrm{~s}^{-1}$ to take into account small pointing errors, and leaving only the amplitude as a completely free parameter.

The minimum $\mathrm{CND}$ emission $\left(\mathrm{CND}_{\min }\right)$ was found by fitting the observed profiles with two additional Gaussians with central velocities fixed at $V_{\mathrm{LSR}}=440 \pm 10 \mathrm{~km} \mathrm{~s}^{-1}$ and $V_{\mathrm{LSR}}=$ $635 \pm 15 \mathrm{~km} \mathrm{~s}^{-1}$ respectively, and leaving both amplitude and velocity width as free parameters. The sum of the two CND Gaussians has a minimum around the systemic velocity, thus maximising the ETD contribution. In the $\mathrm{CND}_{\min }$ decomposition, we find roughly equal amounts of flux for the CND and for the normalized ETD in all transitions (see Table 7). 
Table 7. Decomposed spectral line emission distributions.

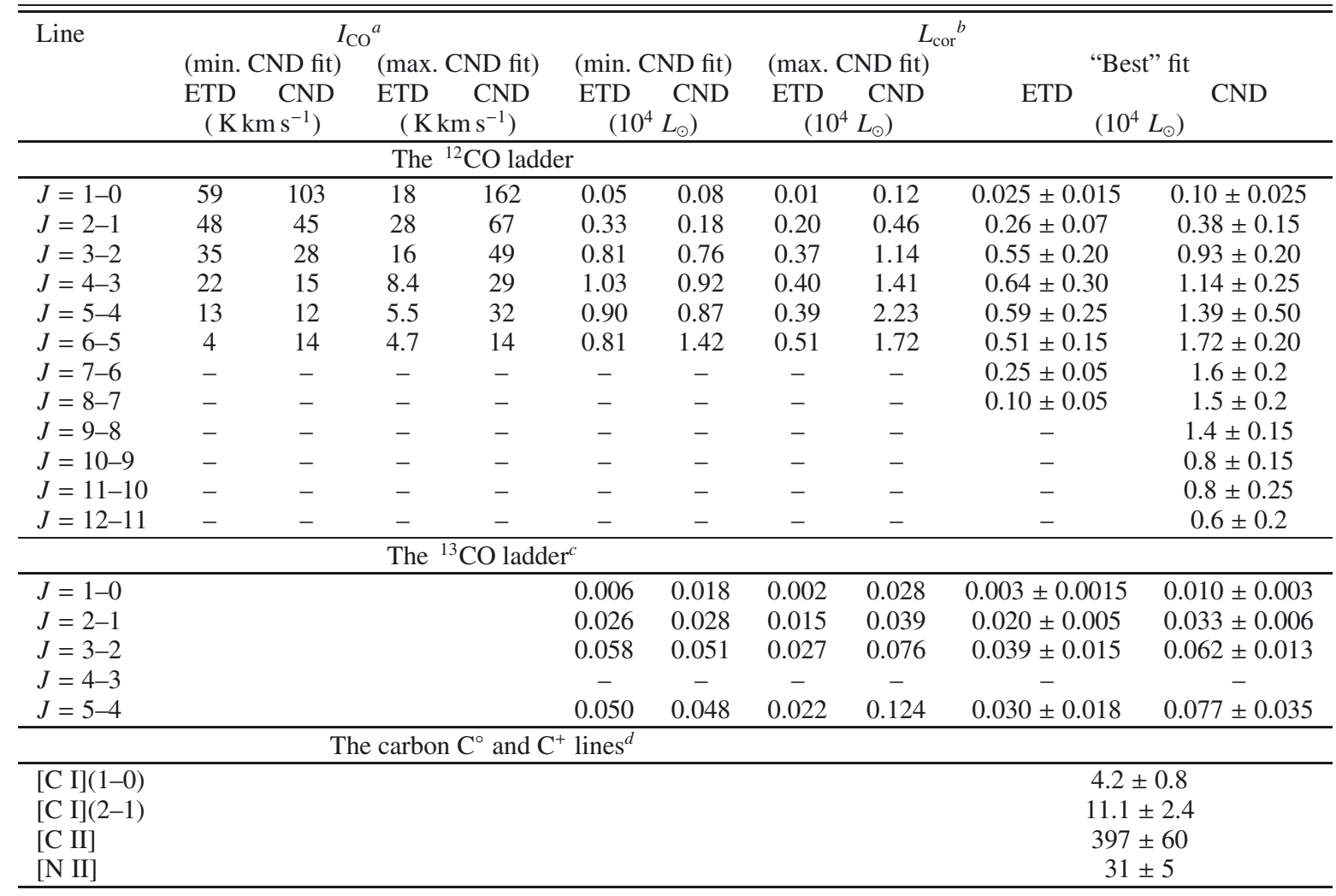

Notes. ${ }^{(a)}$ Emission line intensity normalised to the response of a $22^{\prime \prime}$ beam, corrected for absorption line losses. ${ }^{(b)}$ Adopted emission line luminosity in a $22^{\prime \prime}$ beam, corrected for absorption line losses. ${ }^{(c)}$ Using the appropriate ${ }^{12} \mathrm{CO} /{ }^{13} \mathrm{CO}$ ratios from Table $3 .{ }^{(d)}$ No attempt has been made to decompose these spectra into ETD and CND contributions, but the ETD contribution appears to be weak or even negligible.

The maximum $\mathrm{CND}$ emission $\left(\mathrm{CND}_{\max }\right.$ ) was found by fitting the observed profiles with only one additional Gaussian with free parameters. This second CND Gaussian peaks at roughly the same (systemic) velocity as the first Gaussian representing the ETD contribution, which thus is minimised. Because the CND is not a completely filled disk (cf. Israel et al. 1990), a single Gaussian component fitted to the wings of the observed profile will effectively overestimate the CND contribution. In this decomposition, the ETD contribution in the normalized beam is only a fifth to a fourth of the CND luminosity.

Table 7 summarises the results for both decompositions $\left(\mathrm{CND}_{\min }\right.$ and $\left.\mathrm{CND}_{\max }\right)$ in the $\mathrm{CO}$ transitions up to $J=6-5$. The last two columns contain the mean of these two decompositions which we believe that best represents the actual situation. The CND:ETD flux/luminosity ratio in a $22^{\prime \prime}(410 \mathrm{pc})$ beam is typically $2: 1$ in the lower transitions. Taking into account the beam filling factor of the CND, its mean line surface brightness exceeds that of the ETD by about a factor of four in these transitions.

\subsection{Radiative transfer modelling}

\subsubsection{LVG modelling}

We have modelled the observed ${ }^{12} \mathrm{CO}$ and ${ }^{13} \mathrm{CO}$ line intensities and ratios with the RADEX large velocity gradient (LVG) radiative transfer models (Jansen 1995; Jansen et al. 1994; Hogerheijde \& van der Tak 2000; http://www. strw. leidenuniv.nl/ michiel/ratran/). These codes provide model line intensities as a function of three input parameters per molecular gas phase: molecular gas kinetic temperature $T_{\mathrm{k}}$, density $n\left(\mathrm{H}_{2}\right)$, and the $\mathrm{CO}$ velocity gradient $N(\mathrm{CO}) / \mathrm{d} V$. By comparing model to observed line ratios, we may identify the physical parameters best describing the actual conditions. We present both the result of modelling the observed integral $\mathrm{CO}$ ladder and those of the derived individual CND and ETD CO ladders.

In the modelling, we assume a constant $\mathrm{CO}$ isotopical abundance $\left[{ }^{12} \mathrm{CO}\right] /\left[{ }^{13} \mathrm{CO}\right]=40$ throughout. This value is close to values found in various galaxy centers (Mauersberger \& Henkel 1993; Henkel et al. 1993, 1994, 1998; Bayet et al. 2004). We identify acceptable fits by searching a grid of model parameter combinations $\left(T_{\mathrm{k}}=10-150 \mathrm{~K}, n\left(\mathrm{H}_{2}\right)=10^{2}-10^{5} \mathrm{~cm}^{-3}\right.$, and $N(\mathrm{CO}) / \mathrm{d} V=6 \times 10^{15}-3 \times 10^{18} \mathrm{~cm}^{-2}$ ) for (combined) line ratios matching those observed. In the case of two-phase models, the relative contribution of the two components is treated as a free parameter. The physical gas properties undoubtedly encompass a wider range of temperatures and densities than suggested by even a two-phase model. However, lacking a physical model for the distribution of clouds and their sources of excitation, two phases is the maximum that can be considered fruitfully, especially as only the well-defined lower $J \mathrm{CO}$ transitions have ${ }^{13} \mathrm{CO}$ observations that allow us to break the $T_{\mathrm{k}}-n\left(\mathrm{H}_{2}\right)$ degeneracy inherent to the ${ }^{12} \mathrm{CO}$ ladder.

We find that no combination of parameters from a singlephase gas provides a satisfactory fit to the observed line intensities. This is not unexpected in view of the profile complexity involving the two distinct contributions from ETD and CND. Consequently, we have also modelled the ${ }^{12} \mathrm{CO}$ and ${ }^{13} \mathrm{CO}$ simultaneously with two molecular gas phases. Although the available ${ }^{13} \mathrm{CO}$ measurements break much of the strong 

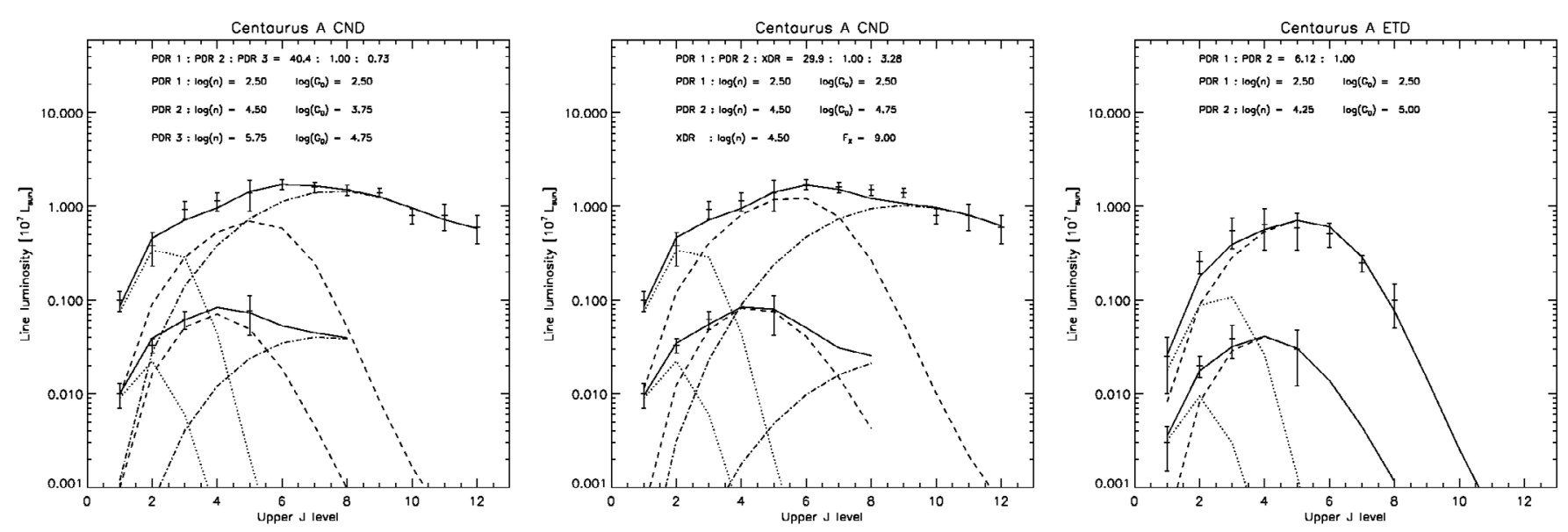

Fig. 5. Results of the PDR/XDR model fitting to the NGC 5128 CO ladders. Left: fit to the CND CO ladder with three PDR models; center: fit to the CND CO ladder with two PDR models and one XDR model; right: fit to the ETD CO ladder with two PDR modelss.

temperature-density degeneracy inherent to ${ }^{12} \mathrm{CO}$ measurements, we still find three different possible solutions.

The first set of solutions puts all gas at an elevated temperature of $T_{\text {kin }}=150 \mathrm{~K}$. In this set, two thirds of the emission comes from a low-density first phase $\left(n\left(\mathrm{H}_{2}\right)=100 \mathrm{~cm}^{-3}\right)$, and one third from a moderate-density second phase $\left(n\left(\mathrm{H}_{2}\right)=1000 \mathrm{~cm}^{-3}\right)$.

In the second set of possible solutions, the two phases have similar modest densities and elevated temperatures. They differ mainly in their $\mathrm{CO}$ velocity gradient $(3 \times$ $10^{16} \mathrm{~cm}^{-2} / \mathrm{km} \mathrm{s}^{-1}$ versus $4.5 \times 10^{17} \mathrm{~cm}^{-2} / \mathrm{km} \mathrm{s}^{-1}$, for the second phase). About $80-90 \%$ of the emission arises from the first phase with kinetic temperatures of 100-150 K, and densities that could be as low as $100 \mathrm{~cm}^{-3}$ or as high as $1000 \mathrm{~cm}^{-3}$, but are most likely around $500 \mathrm{~cm}^{-3}$. The remaining $20-10 \%$ arises a slightly warmer, and slightly more tenuous second phase $\left(T_{\text {kin }} \approx 150 \mathrm{~K}, n\left(\mathrm{H}_{2}\right)=100-500 \mathrm{~cm}^{-3}\right)$.

The final set of solutions combines an again very similar first phase $\left(T_{\text {kin }}=100 \mathrm{~K}, n\left(\mathrm{H}_{2}\right)=500 \mathrm{~cm}^{-3}\right)$, with a much denser and colder second phase $\left(T_{\text {kin }}=20-30 \mathrm{~K}, n\left(\mathrm{H}_{2}\right)=10^{5} \mathrm{~cm}^{-3}\right)$.

We emphasize that the different physical environments represented by the solutions are observationally indistinguishable. Moreover, they are not mutually exclusive but may apply simultaneously and sample a multi-phase structure of the ISM: very dense, cold clumps embedded in a more tenuous warm molecular gas.

Thus, the LVG analysis of the integral line profile requires the presence of significant amounts of warm gas at temperatures of $100-150 \mathrm{~K}$ at moderate densities of typically $500 \mathrm{~cm}^{-3}$, but does not distinguish between the situation where this is all, where two thirds of the emission is from a more tenuous warm gas $\left(100 \mathrm{~cm}^{-3}\right)$, or where $10-15 \%$ of the emission comes from a much more dense, cold gas. As it turns out, only the latter case fulfils the additional constraints posed by considering the CND and the ETD separately.

When we fit LVG models to the individual CND and ETD components, once again no single phase model provides a reasonable fit to the observed CO line ladders. However, the CND $\mathrm{CO}$ ladder is well-fitted by two gas phase components in a narrow range, involving a dense $\left(n_{\mathrm{H}_{2}}=10^{4} \mathrm{~cm}^{-3}\right)$ and a much more tenuous $\left(n_{\mathrm{H}_{2}}=300 \pm 200 \mathrm{~cm}^{-3}\right)$ gas. The properties of the dense gas are well-defined, with a low temperature $T_{\text {kin }}=25 \pm 5$. The temperature of the tenuous gas temperature is not so tightly constrained. It may be at $T_{\text {kin }}=60-100 \mathrm{~K}$, in which case it will be responsible for three quarters of the $\mathrm{CO}$ emission in the $J=2-1$ transition, but it may also be at a much lower $T_{\text {kin }}=20-30 \mathrm{~K}$ in which case its $J=2-1$ emission share will be about $50-60 \%$.

The physical parameters of the ETD gas are better determined and differ markedly from those of the CND gas. Most $(65 \pm 15 \%)$ of the emission in the $J=2-1$ transition is from a gas with a well-established high density $\left(n_{\mathrm{H}_{2}}=10^{4} \mathrm{~cm}^{-3}\right)$ and low temperature $T_{\text {kin }}=30 \mathrm{~K}$. The remainder of the gas (responsible for $35 \pm 15 \%$ of the emission) has a very low density $\left(n_{\mathrm{H}_{2}}=100 \mathrm{~cm}^{-3}\right)$ at an elevated temperature $T_{\text {kin }}=100 \pm 50 \mathrm{~K}$.

\subsubsection{PDR/XDR modelling}

To further investigate the physical conditions and excitation mechanisms, we have also applied the PDR/XDR models by Meijerink \& Spaans (2005) and Meijerink et al. (2007). In XDRs, the excitation is dominated by $\mathrm{X}$-ray photo-ionization heating, i.e., the Coulomb interaction of $\mathrm{keV}$ electrons with thermal electrons. The heating efficiency of X-rays is of the order of 10 to 40 percent, much more efficient than the photo-electric heating ( $\sim 0.3$ to 1.0 percent) in PDRs. The ionisation is driven by primary and secondary X-ray ionisations, and the ionisation fraction can be larger than $x_{\mathrm{e}} \sim 0.1$, three orders of magnitude higher than in PDRs. The high degree of ionisation is able to drive an active ion-molecule chemistry and makes it possible to maintain high abundance levels of molecules at high temperatures, $T>300 \mathrm{~K}$. This expresses itself in much larger column densities of warm gas in XDRs, which betray themselves by much more intense higher $J$ CO transitions.

The PDR/XDR code requires three input parameters: a density $n$, a surface area covering factor (in this case relative to that of component PDR2), and an incident UV flux in units of the one-dimensional Habing (1968) field $G_{0}(=1.6 \times$ $10^{-3}$ erg $\mathrm{cm}^{-2} \mathrm{~s}^{1}$ ), or an X-ray energy flux $F_{\mathrm{X}}$ (in units of $\mathrm{cm}^{-2} \mathrm{~s}^{1}$ ), respectively. We constrained the PDR parameters to be fitted by using the relatively well-established gas volume densities supplied by the LVG modelling for the first two gas components. The resulting fits are shown in Fig. 5. The boxes on the left and in the center show fits to the CO ladder of the CND using three PDRs, and two PDRs and one XDR, respectively. The box on the right e shows the fit to the $\mathrm{CO}$ ladder of the ETD in the same line of sight, which requires only two PDRs. Throughout, the major contributor to the low- $J$ emission of both ${ }^{12} \mathrm{CO}$ and ${ }^{13} \mathrm{CO}$ (PDR1) is well-represented by the 
Table 8. CND model physical parameters.

\begin{tabular}{|c|c|c|c|c|}
\hline & \multicolumn{2}{|c|}{ LVG model } & \multicolumn{2}{|c|}{ PDR model } \\
\hline & CND & ETD & CND & ETD \\
\hline \multicolumn{5}{|c|}{ ISM phase 1} \\
\hline Density $n\left(\mathrm{H}_{2}\right)\left(\mathrm{cm}^{-3}\right)$ & 300 & 100 & 300 & 300 \\
\hline Temperature $T_{\mathrm{k}}(\mathrm{K})$ & $25-80$ & 100 & - & - \\
\hline UV radiation field $G\left(G_{0}\right)$ & - & - & 300 & 300 \\
\hline Column density $N_{\mathrm{H}}\left(10^{21} \mathrm{~cm}^{-2}\right)$ & $5-10$ & & 13 & \\
\hline \multicolumn{5}{|c|}{ ISM phase 2} \\
\hline Density $n\left(\mathrm{H}_{2}\right)\left(\mathrm{cm}^{-3}\right)$ & 10000 & 10000 & 30000 & 18000 \\
\hline Temperature $T_{\mathrm{k}}(\mathrm{K})$ & 25 & 30 & - & - \\
\hline UV radiation field $G\left(G_{0}\right)$ & - & - & $3000-30000$ & 10000 \\
\hline Column density $N_{\mathrm{H}}\left(10^{21} \mathrm{~cm}^{-2}\right)$ & $2-4$ & & 13 & \\
\hline \multicolumn{5}{|c|}{ Either ISM phase 3: PDR } \\
\hline Density $n\left(\mathrm{H}_{2}\right)\left(\mathrm{cm}^{-3}\right)$ & - & - & 550000 & \\
\hline UV radiation field $G\left(G_{0}\right)$ & - & - & 55000 & \\
\hline Column density $N_{\mathrm{H}}\left(10^{21} \mathrm{~cm}^{-2}\right)$ & - & - & 7 & \\
\hline \multicolumn{5}{|c|}{ Or ISM phase 3: XDR } \\
\hline Density $n\left(\mathrm{H}_{2}\right)\left(\mathrm{cm}^{-3}\right)$ & - & - & 30000 & \\
\hline X-ray flux $F_{\mathrm{x}}\left(\mathrm{erg} \mathrm{cm}^{-2} \mathrm{~s}^{-1}\right)$ & - & - & $5-16$ & \\
\hline Column density $N_{\mathrm{H}}\left(10^{21} \mathrm{~cm}^{-2}\right)$ & - & - & $10-100$ & \\
\hline
\end{tabular}

selected density $n=300 \mathrm{~cm}^{-3}$ and a moderate incident radiation field $G=300 G_{0}$. It has a large surface filling factor compared to PDR2, the component dominating the mid- $J$ transitions $(3 \leq J<8)$. PDR 2 requires a more intense incident radiation field $G=10^{3.75}-10^{4.75} G_{0}$ with higher densities $n=3 \times 10^{4} c c$ for the CND and $n=10^{4} c c$ for the ETD. Its relatively small surface filling factor implies that the dense, strongly irradiated component exists mostly or entirely in clumped form. We note that the fits are not unique, and the ones presented here are merely those with the lowest chi-square values. Especially the radiation field is not well-constrained. The CO emission emitted by PDRs is not very sensitive to the radiation field, as it originates mostly from the UV-shielded part of the clouds.

The third component is a significant contributor only in the high $J$ transitions $(J \geq 8)$. Its existence is required by the CND CO ladder which reliably extends to the higher $J$ transitions (Sect. 4.3). This high-energy component represents either extreme PDR conditions (PDR3), or X-ray irradiation (XDR). The extreme PDR case must have very high densities of the order of $n=10^{6} \mathrm{~cm}^{-3}$ (consistent with the detection of HCN emission from the CND) and radiation fields $G=55000 G_{0}$. It has a slightly smaller filling factor than that of PDR2, and also has a three times lower total column density, $N_{\mathrm{H}} \sim 7.3 \times 10^{-21} \mathrm{~cm}^{-2}$. On the other hand, if it represents an XDR, the density does not need to exceed that of PDR2 $\left(n=10^{4.5} \mathrm{~cm}^{-3}\right)$, with an X-ray radiation field of $F=9.0 \mathrm{erg} \mathrm{cm}^{2} \mathrm{~s}^{1}$ and a column density of $N_{\mathrm{H}}=3 \times 10^{22} \mathrm{~cm}^{-2}$. In that case, its surface filling factor is a few times than that of PDR2 (but still much less than that of PDR1). Equally good fits are, however, obtained for XDR phases with column densities ranging from $N_{\mathrm{H}}=10^{22}$ to $10^{23} \mathrm{~cm}^{-2}$, and concomitant X-ray fluxes of $F_{\mathrm{X}}$ of 5 and $16 \mathrm{erg} \mathrm{cm}^{-2} \mathrm{~s}^{-1}$. respectively. As Fig. 5 shows, the observational data do not significantly constrain the CND physics because both PDR3 and (a variety of) XDR models provide comparably good fits to the $\mathrm{CO}$ ladder. Observationally, the appropriate models can only be identified by CO line intensities at e.g. the $J=15-14$ transition and beyond which are presently lacking.

\subsection{Beam-averaged molecular gas properties}

In order to derive overall molecular gas column densities from the LVG data, we assume that only about a quarter of all carbon is in the gas-phase $\left(\delta_{\mathrm{C}}=0.27\right)$, the remainder being tied down in dust grains. In a forthcoming paper, we find that the ISM of both the CND and the ETD is characterized by a metalicity of about 0.7-0.8 times that of the Solar Neighbourhood. We assume a $[\mathrm{C}] /[\mathrm{H}]$ elemental abundance $x_{\mathrm{c}}=1.6 \times 10^{-4}$ which leads us to expect expect a neutral gas-phase $N_{\mathrm{C}} / N_{\mathrm{H}}=4.5 \times 10^{-5}$. The chemical models by van Dishoeck \& Black (1988), updated by Visser et al. (2009) show a strong dependence of the ratio of atomic carbon to molecular carbon monoxide column densities on the total carbon $N_{\mathrm{C}}=N(\mathrm{C})+\mathrm{N}(\mathrm{CO})$ and molecular hydrogen $N\left(\mathrm{H}_{2}\right)$ column densities. Thus, each value of $N\left(\mathrm{H}_{2}\right)$ is associated with a unique value of $N(\mathrm{C}) / \mathrm{N}(\mathrm{CO})$ and a unique value of $N_{\mathrm{C}}=$ $N(\mathrm{C})+\mathrm{N}(\mathrm{CO})$ which can be derived from the models.

From the LVG analysis, we find very similar beam-averaged column densities for the CND $\left(N(\mathrm{CO})=0.34 \times 10^{18}\right)$ and for the ETD $\left(N(C O)=0.29 \times 10^{18} \mathrm{~cm}^{-2}\right)$. We also find that in both the CND and the ETD about two to three times more carbon is in atomic than in molecular form, and we obtain a beam-averaged total hydrogen column $N_{\mathrm{H}}=(3.4 \pm 0.1) \times 10^{22} \mathrm{~cm}^{-2}$ for the CND, and $N_{\mathrm{H}}=(1.0 \pm 0.1) \times 10^{22} \mathrm{~cm}^{-2}$ for the ETD. Taking into account that the ETD fills all of the normalized beam, and the CND only half of it, we derive a four times higher molecular gas surface-filling factor $(0.10 \pm 0.01)$ for the CND.

The molecular gas column density is found by subtracting the neutral hydrogen $\mathrm{H}$ I from the total hydrogen column density. Measurements of the H I emission suggest its contribution is quite small in the central few hundred parsecs (see Struve et al. 2010). On the other hand, H I absorption line measurements imply very high $\mathrm{H}$ I column densities (typically $10^{22}-10^{23} \mathrm{~cm}^{-2}$ ), but these apply to a pencil-beam area of order $10^{5}$ smaller and are related to material much less extended than the scale of the CND. Neglecting a possible (but probably small) contribution by atomic hydrogen, we find for the CND a beam-averaged molecular hydrogen column density $N\left(\mathrm{H}_{2}\right)=(1.7 \pm 0.1) \times 10^{22} \mathrm{~cm}^{-2}$. The corresponding mass is $M_{\text {gas }}=4.7 \pm 0.510^{7} M_{\odot}$, including a $35 \%$ helium contribution. In the ETD the $\mathrm{H}$ I column may not be negligible. For $N(\mathrm{HI}) \leq 0.15 \times 10^{22} \mathrm{~cm}^{-2}$ (cf. Struve et al. 2010) we obtain for the ETD $N\left(\mathrm{H}_{2}\right)=0.9 \times 10^{22} \mathrm{~cm}^{-2}$.

The PDR model analysis produces higher gas masses. The pure PDR-model yields a total gas mass $M_{\text {gas }}=1.5 \times 10^{8} M_{\odot}$ which includes a $35 \%$ contribution of helium. A fraction of 0.95 of this mass resides in the lowest-excitation phase (PDR1), whereas the densest, highest-excitation gas (PDR3) mass is of the order of a per cent of the total. If we assume that the highest observed CO $J$ transitions come from gas excited by X-rays rather than by UV-photons, the mass of this phase can be substantially higher. For instance, if we assume an XDR density identical the PDR2 gas, we obtain a (helium-corrected) gas mass $M_{\text {gas }}=1.7 \times 10^{8} M_{\odot}$, of which $15 \%$ is in the X-ray irradiated phase. This total gas mass is only slightly higher than that of the pure PDR case. In the fraction of the ETD measured by our beam, the mass density is no more than a quarter of that of the CND, and only $15 \%$ is in a denser, more excited phase (PDR2). As shown in Fig. 5, there is no third phase of very dense gas highly excited by X-rays or their equivalent. In the pure PDR case, the mass of dense gas in the CND is not much higher than that in the ETD-beam. However, in the XDR case the mass of dense gas is ten times higher in the CND than in the ETD-beam. This is in better agreement with the observation that 
Table 9. NGC 5128 CND properties.

\begin{tabular}{|c|c|}
\hline Projected dimensions (pc) & $390 \times 195$ \\
\hline Velocity width FWHM $\Delta V\left(\mathrm{~km} \mathrm{~s}^{-1}\right)$ & 375 \\
\hline Surface area filling factor & $0.10 \pm 0.02^{a}$ \\
\hline Mean column density ${ }^{a} N_{\mathrm{H}}\left(\mathrm{cm}^{-2}\right)$ & $(0.3-1.5) \times 10^{23}$ \\
\hline $\operatorname{Gas} \operatorname{mass}^{b, c} M_{\text {gas }}\left(M_{\odot}\right)$ & $8.4 \times 10^{7}$ \\
\hline Conversion factor ${ }^{d} X\left(\mathrm{~cm}^{-2} / \mathrm{K} \mathrm{km} \mathrm{s}^{-1}\right)$ & $4 \times 10^{20}$ \\
\hline CO luminosity ${ }^{e} L_{\mathrm{CO}}\left(L_{-} \odot\right)$ & $(3.6-5.0) \times 10^{5}$ \\
\hline $\mathrm{C}^{\circ}$ luminosity $L_{\mathrm{CI}}\left(L_{-} \odot\right.$ & $(1.5 \pm 0.3) \times 10^{5}$ \\
\hline $\mathrm{C}^{+}$luminosity $L_{\mathrm{CII}}\left(L_{-} \odot\right.$ & $(4.0 \pm 0.6) \times 10^{6}$ \\
\hline
\end{tabular}

Notes. ${ }^{(a)}$ LVG two-phase and PDR three-phase result; uncertainty $50 \%$. (b) Uncertainty factor of three; lower limit from LVG assuming all $\mathrm{H}$ in $\mathrm{H}_{2}$, upper limit from PDR/XDR modelling assuming no mechanical heating in PDR1. ${ }^{(c)}$ Assuming a 35\% mass contribution by helium. ${ }^{(d)}$ Uncertainty factor of two. ${ }^{(e)}$ Sum of all CO transitions up to $J=15-14$.

the HCN emission (Table 3 and Fig. 4) traces dense gas almost exclusively in the CND.

In the PDR/XDR models the atomic gas mass fraction ranges between one-third to half of the total gas mass. This is because quite large radiation fields are needed to obtain PDR excitation conditions that are able to reproduce the observed line fluxes. This can, however, be mitigated by including a small amount of mechanical heating (cf. Kazandjian et al. 2012, 2013). When sloshing motions of the gas are responsible for part of the total heating, especially in the regions where the gas is shielded from UV radiation, the incident UV fluxes can be substantially lowered (yielding a smaller atomic hydrogen fraction), while obtaining the same $\mathrm{CO}$ model fluxes.

The physical properties of the NGC 5128/Centaurus A circumnuclear disk are summarised in Table 9. For the best estimate of the CND mass we use the geometric mean of the LVG and PDR results. This brings the mass to $M_{\text {gas }}=8.4 \times$ $10^{7} M_{\odot}$ with a corresponding $N\left(\mathrm{H}_{2}\right)$-to- $I^{12} \mathrm{CO}$ ratio $X=4 \times$ $10^{20} \mathrm{~cm}^{-2} / \mathrm{K} \mathrm{km} \mathrm{s}^{-1}$, about twice the "standard" Milky Way ratio. We cannot determine a useful mass value for the small fraction of the ETD included in our beams, but from our analysis we estimate an $X$-value not very different, and possibly a bit closer to that of the Milky Way. This is the first time the CND gas mass has been determined rather than guessed. For instance, a much lower CND mass was given by Espada et al. (2009), but this was based entirely on an assumed very low $X$-factor of $0.4 X_{\mathrm{GAL}}$. This appears to be appropriate for "normal" AGNs, but does not adequately describe the Cen A situation.

In Table 9 we also compare the integrated CO luminosity (using the models to extrapolate the observed CO line luminosities up to the $J=15-14$ transition) with the observed neutral and ionized carbon luminosities. In case of significant X-ray excitation, the total CO luminosity may be somewhat higher than given here, because of the contribution of the very high- $J$ transitions. Nevertheless, from the comparison in Table 9 we may conclude that neutral carbon cooling of the CND is at most half of that provided by carbon monoxide. In contrast, the cooling by ionized carbon is about an order of magnitude higher than the CO cooling.

\section{Conclusions}

1. We have measured ${ }^{12} \mathrm{CO}$ fluxes from the central region of NGC 5128 (Centaurus A) in transitions up to $J=$ $12-11$, as well as the fluxes from the submillimeter [C I] and [C II] lines. In addition, we have presented high-S/N velocity-resolved profiles of ${ }^{12} \mathrm{CO}$ emission in the $J=$ $1-0$ through $J=5-4$ and of ${ }^{13} \mathrm{CO}$ emission in the $J=$ $1-0$ through $J=3-2$ transitions.

2. ${ }^{12} \mathrm{CO}$ line luminosities, normalized to a $22^{\prime \prime}$ beam $(410 \mathrm{pc})$ increase up to $J=5-4$, and then steadily drop to low values. This drop is more pronounced than that seen in Seyfert AGNs, and much more pronounced than seen in star-burst galaxies.

3. Both [C I] lines are more luminous than the adjacent $(J=$ $4-3$, and $J=7-6){ }^{12} \mathrm{CO}$ lines. This behaviour is not seen in other (star-burst or AGN) galaxies, and is thus unique to the Cen A center. The [C I] line intensities and their ratio to $\mathrm{CO}$ line intensities are inconsistent with standard PDR model predictions.

4. Detailed analysis shows that up to $J=6-5$, about a third of the ${ }^{12} \mathrm{CO}$ flux observed in the normalised $22^{\prime \prime}$ beam is contributed by the ETD embedded in NGC 5128, and unrelated to the compact CND just contained within the normalised beam. The CND contains a high proportion of very dense gas, in contrast to the ETD. At transitions above $J=$ $6-5$, the ${ }^{12} \mathrm{CO}$ emission is completely dominated by the CND contribution.

5. We have decomposed the observed $\mathrm{CO}$ spectral line ladder into individual (total) $\mathrm{CND}$ and (representative) ETD CO ladders. The ETD ladder peaks in the $J=4-3 / J=5-4$ transition and then drops rapidly. The CND ladder peaks in the $J=6-5$ and $J=7-6$ transitions before dropping more slowly.

6. $\mathrm{LVG}$ and PDR/XDR model analysis of the CND ${ }^{12} \mathrm{CO}$ fluxes shows that most of the molecular gas mass resides in a relatively cool $\left(T_{\text {kin }}=25-80 \mathrm{~K}\right)$, not very dense $\left(n_{\mathrm{H}_{2}} \approx\right.$ $300 \mathrm{~cm}^{-3}$ ) gas phase if the CND is heated exclusively by UV photons (PDR).

7. A small fraction of the gas in the CND is more highly excited and has much higher densities (typically $3 \times 10^{4} \mathrm{~cm}^{-3}$ ).

8. In the CND but not in the ETD a third, more highly excited, high-density phase must also be present, either in the form of an extreme PDR or in the form of an XDR.

9. The CND has a total gas mass $M_{\mathrm{CND}}=8.4 \times 10^{7} M_{\odot}$ (uncertain by a factor of two) which is about $10 \%$ of the mass of the much larger ETD.

10. The $\mathrm{CO}-\mathrm{H}_{2}$ conversion factor $X_{\mathrm{CND}}$ is $4 \times 10^{20} \mathrm{~K} \mathrm{~km} \mathrm{~s}^{-1}$ (uncertain by a factor of two), about twice the local Milky Way factor $X_{\mathrm{SN}}$.

Acknowledgements. We thank Markus Schmalzl for help with the ALMA SV data. We also thank all facility observers, engineers, and support scientists who by their often anonymous efforts have made possible the collection of data presented in this paper.

\section{References}

Bayet, E., Gerin, M., Phillips, T. G., \& Contursi, A. 2004, A\&A, 427, 45 Booth, R. S., Delgado, G., Hagstrom, M., et al. 1989, A\&A, 216, 31 Cappellari, M., Neumayer, N., Reunanen, J., et al. 2009, MNRAS, 394, 660 de Graauw, Th., Helmich, F. P., Phillips T. G., et al. 2010, A\&A, 518, L6 Dufour, R. J., Harvel, C. A., Martins, D. M., et al. 1979, AJ, 84, 284 Espada, D., Matsushita, S., Peck, A., et al. 2009, ApJ, 695, 116 Fixsen, D. J., Bennett, C. L., \& Mather, J. C. 1999 ApJ, 526, 207 Graham, J. A. 1979, ApJ, 232, 60

Griffin, M. J., Abergel, A., Abreu, A., et al. 2010, A\&A, 518, L3 Güsten, R., Nyman, L.-A., Schilke, P., et al. 2006, A\&A, 454, L13 Güsten, R., Baryshev, A., Bell, A., et al. 2008, SPIE, 7020, 25 Habing, H. J. 1968, Bull. Astron. Inst. Netherland, 19, 421

Hawarden, T. G., Sandell, G., Matthews, H. E., et al. 1993, MNRAS, 260, 844 Henkel, C., \& Wiklind T. 1997, Space Sci. Rev., 81, 1 
F. P. Israel et al.: Cen A circumnuclear disk

Henkel, C., Mauersberger, R., Wiklind, T., et al. 1993, A\&A, 268, L17 Henkel, C., Whiteoak, J. B., \& Mauersberger, R. 1994 A\&A, 284, 17 Henkel, C., Chin, Y.-N, Mauersberger, R., \& Whiteoak, J. B. 1998, A\&A, 329, 443

Heyminck, S., Kasemann, C., Güsten, R., de Lange, G., \& Graf, U. U. 2006, A\&A, 454, L21

Hitschfeld, M., Aravena, M., Kramer, C., et al. 2008, A\&A, 479, 75

Hogerheijde, M. R., \& van der Tak, F. F. S. 2000, A\&A, 362, 697

Israel, F. P. 1992, A\&A, 265, 487

Israel, F. P. 1998, A\&ARv, 8, 237

Israel, F. P. 2005, Ap\&SS, 295, 171

Israel, F. P., \& Baas, F. 2002, A\&A, 383, 82

Israel, F. P., van Dishoeck, E. F., Baas, F., et al. 1990, A\&A, 227, 342

Israel, F. P., van Dishoeck, E. F., Baas, F., de Graauw, T., \& Phillips, T. G. 1991, A\&A, 245, L13

Israel, F. P., Raban, D., Booth, R. S., \& Rantakyrö, F. T. 2008, A\&A, 483, 741

Jansen, D. J. 1995, Ph.D. Thesis, University of Leiden (NL)

Jansen, D. J., van Dishoeck, E. F., \& Black, J. H. 1994, A\&A, 282, 605

Kasemann, C., Güsten, R., Heyminck, S., et al. 2006, SPIE, 6275, 19

Kazandjian, M. V., Meijerink, R., Pelupessy, I., Israel, F. P., \& Spaans, M. 2012, A\&A, 542, A65

Kazandjian, M. V., Meijerink, R., Pelupessy, I., Israel, F. P., \& Spaans, M. 2013, A\&A, submitted
Klein, B., Philipp, S. D., Krämer, I., Kasemann, C., Güsten, R., \& Menten, K. M. 2006, A\&A, 454, L29

Liszt, H. 2001, A\&A, 371, 865

Mauersberger, R., \& Henkel, C. 1993, Rev. Mod. Astron., 6, 69

Meijerink, R., \& Spaans, M. 2005, A\&A, 436, 397

Meijerink, R., Spaans, M., \& Israel, F. P. 2007, A\&A, 461, 793

Meijerink, R., Kristensen, L. E., Weiss, A., et al. 2013, ApJ, 762, L16

Meisenheimer, K., Tristram, K. R. W., Jaffe, W., et al. 2007, A\&A, 471, 453

Nicholson, R. A., Bland-Hawthorn, J., \& Taylor, K. 1992, ApJ, 387, 503

Panuzzo, P., Rangwala, N., Rykala, A., et al. 2010, A\&A, 518, L37

Papadopoulos, P. P., Zhang, Z.-Y., Weiss, A., et al. 2013, ApJ submitted

Pereira-Santaella, M., Spinoglio, L., Busquet, G., et al. 2013, ApJ, 768, 55

Pilbratt, G. L., Riedinger, J. R., Passvogel, T., et al. 2010, A\&A, 518, L1

Rangwala, N., Maloney, P. R., Glenn, J., et al. 2011, ApJ, 743, 94

Rydbeck, G., Wiklind, T., Cameron, M., et al. 1993, A\&A, 270, L13

Spaans, M., \& Meijerink, R. 2008, ApJ, 678, L5

Spinoglio, L., Pereira-Santaella, M., Busquet, G., et al. 2012, ApJ, 758, 108

Struve, C., Oosterloo, T. A., Morganti, R., \& Saripalli, L. 2010, A\&A, 515, A67

Unger, S. J., Clegg, P. E., Stacey, G. J., et al. 2000, A\&A, 355, 885

van der Werf, P. P., Isaak, K. G., Meijerink, R., et al. 2010 A\&A, 518, L42

van Dishoeck, E. F., \& Black, J. H. 1988, ApJ, 334, 771

Visser, R., Van Dishoeck, E. F., \& Black, J. H. 2009, A\&A, 503, 323

Wild, W., Eckart, A., \& Wiklind, T. 1997, A\&A, 322, 419 